\title{
L'évolution des nappes alluviales antérissiennes de la Garonne,
} dans l'avant-pays molassique

Jacques Hubschman

\section{Abstract}

Description and analysis of the Garonne pre-Riss deposits. Mindel alluvial pebble weathering is shown by : strong weathering of crystalline material ; clay formation and argillic development ; genesis of kaolinic minerals but very disorderly ; water-tighting of pebble alluvions ... Gunz materials are little more weathered but Donau alluvions are extremly weathered, with important amounts of well crystallized kaolinic minerals. Iron-crust genesis and origin of silt cover are discussed.

\section{Citer ce document / Cite this document :}

Hubschman Jacques. L'évolution des nappes alluviales antérissiennes de la Garonne, dans l'avant-pays molassique. In: Bulletin de l'Association française pour l'étude du quaternaire, vol. 12, n³-4, 1975. pp. 149-169;

doi : https://doi.org/10.3406/quate.1975.1262

https://www.persee.fr/doc/quate_0004-5500_1975_num_12_3_1262

Fichier pdf généré le 19/04/2018 


\section{L'ÉVOLUTION DES NAPPES ALLUVIALES ANTERISSIENNES DE LA GARONNE, DANS L'AVANT-PAYS MOLASSIQUE \\ par Jacques HUBSCHMAN}

\section{SUMMARY}

Description and analysis of the Garonne pre-Riss deposits. Mindel alluvial pebble weathering is shown by : strong weathering of crystalline material; clay formation and argillic development; genesis of kaolinic minerals but very disorderly; water-tighting of pebble alluvions... Günz materials are little more weathered but Donau alluvions are extremly weathered, with important amounts of well crystallized kaolinic minerals. Iron-crust genesis and origin of silt cover are discussed.

\section{I. - LA TERRASSE MINDELIENNE : EVOLUTION PEDO-GEOCHIMIQUE (*)}

Bien conservée dans son ensemble, la terrasse moyenne de la Garonne s'étale largement et de manière continue depuis les Petites Pyrénées jusqu'au confluent tarnais (fig. 9). Le talus qui la limite vers l'est est généralement bien marqué dans le paysage, au-dessus de la basse terrasse rissienne. En revanche, le talus occidental, que domine la nappe günzienne, est loin d'être toujours aussi net : d'où la difficulté, parfois, de séparer clairement ces deux générations alluviales, notamment entre Save et Gimone.

Une première constatation s'impose, à propos de la différenciation pédologique des couvertures limoneuses. Alors que, du Würm au Riss, les profils développés dans les limons passent nettement d'un modèle "brun lessivê" à un modèle "lessivé hydromorphe", le passage du Riss au Mindel ne se traduit pas vraiment par une accentuation très sensible de la différenciation, aussi bien sur le plan macroscopique et morphologique qu'au niveau analytique. Certes, quelques faciès, encore peu répandus dans les limons rissiens et liés à la dégradation hydromorphe, comme les glosses, prennent à partir du Mindel une plus large extension. Mais, à cette réserve près - et si l'on exclut les horizons à grep, dont la genèse est particulière -, l'évolution des limons de la terrasse rissienne ne diffère pas fondamentalement de celle qui marque le niveau mindélien (et également les alluvions plus anciennes). On touche ici au problème de l'origine et de l'âge de ces couvertures limoneuses qui drapent très généralement les terrasses alluviales du piémont nord-pyrénéen : on reviendra sur cette question à propos de la nappe culminante garonnaise et dans les dernières pages de cet exposé.

L'altération des alluvions caillouteuses mindéliennes, en revanche, change distinctement de degré et de nature. La coupe de l'arrêt $\mathrm{n}^{\circ} 10$, située au sommet du talus dominant la terrasse rissienne, permet précisément d'observer un profil tronqué et rajeuni dans les cailloutis mindéliens, qui contraste déjà singulièrement avec les profils rissiens. Mais c'est surtout l'arrêt $n^{\circ} 11$ qui a valeur de modèle pour l'ensemble des remblaiements grossiers de la nappe mindélienne. La carrière, ouverte sur le rebord droit de la vallée de l'Aussonnelle, recoupe toute la tranche d'alluvions, ici peu épaisses (4 $\mathrm{m}$ environ) et s'enfonce dans la molàsse exploitée par la briqueterie Gélis (photo 13).

(*) Pour plus de commodité dans la présentation, on a choisi de ne pas respecter exactement l'itinéraire de l'excursion $\left(2^{\mathrm{e}}\right.$ journéc), dont le premier arrêt concernait un faciès de nappe culminante. 


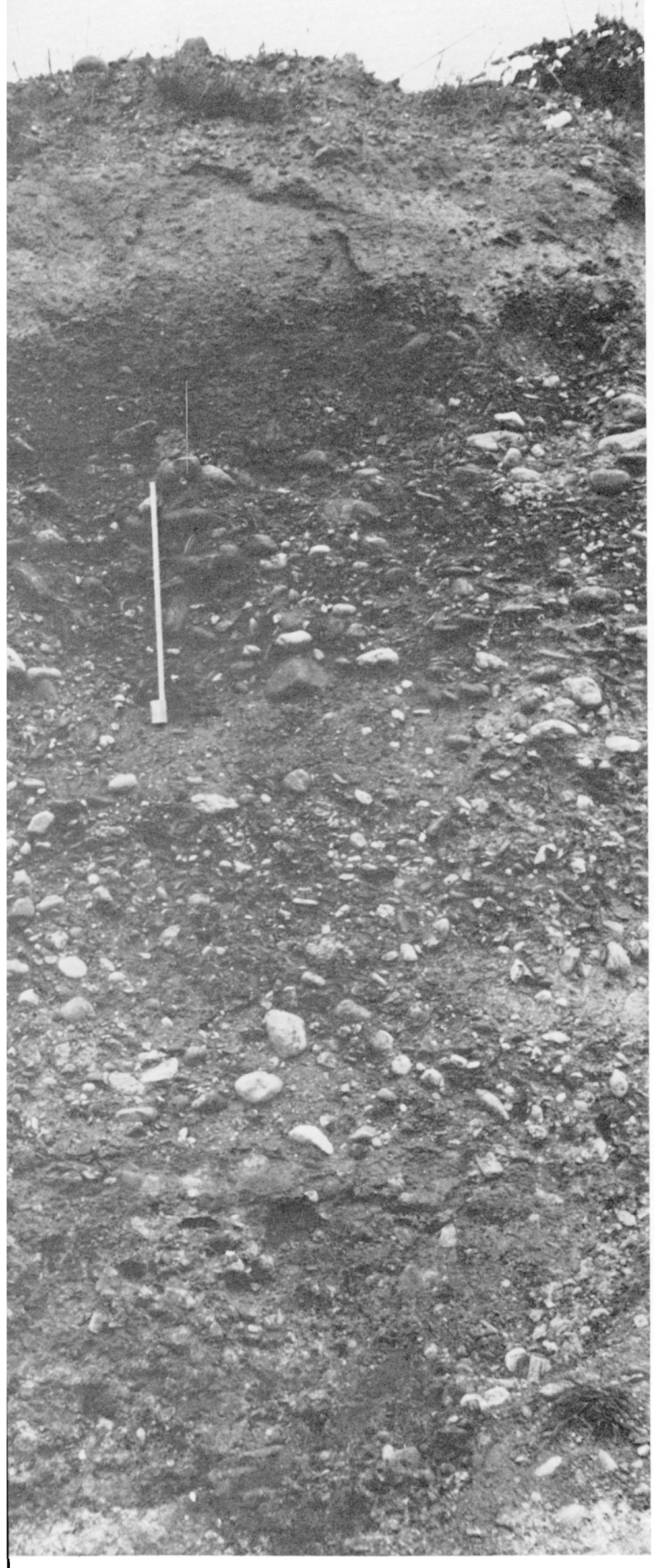

\section{Description sommaire du profil} de l'arrêt $n^{\circ} 11$ :

Sol lessivé hydromorphe, profil

d'altération à tendance fersiallitique

- $0-30 \mathrm{~cm}$ : Ap, très remanié à débris de molasse (marnage), tassé, structure massive, graviers et gravillons en amas, TRA $10 \%$, racines, limite inférieure graduelle et ondulée, par place franche et rectiligne;

$-30-65 \mathrm{~cm}: A_{2}$ et $B_{1 \mathrm{tg}}, 10$ YR $5 / 4$, structure massive à tendance lamellaire, polyédrique en profondeur, graviers et galets TRA 10 à $15 \%$ patinés et sombres, macropores abondants, concrétions rondes et noires peu nombreuses et enduits noirs des graviers en profondeur, limite inférieure franche, très ondulée;

$-65-80 \mathrm{~cm}: \mathrm{B}_{2 \mathrm{tg}}$, limon riche en gravillons, graviers et quelques galets TRA 30 à $50 \%$, fond 10 YR $5 / 4$ avec plages 10 YR $3 / 1$ des enduits et concrétions noirs abondants passant localement et latéralement à des faciès de grep tendre. Structure massive, sousstructure polyédrique, revêtements argileux, limite inférieure assez franche et ondulée ;

- 80-130 cm: II $B_{3 t \mathrm{~g}}$, nappe caillouteuse TRA 50 à $70 \%$, matrice bigarrée fond 10 YR $5 / 6$ à 5 YR $5 / 6$ à $5 / 8$, avec plages $5 \mathrm{Y} 5 / 2$ en bandes subhorizontales et plages noires des enduits et concrétions, épais revêtements argileux, éléments cristallins très altérés parfois totalement intégrés à la matrice, limite inférieure graduelle et ondulée ;

$-130-220 \mathrm{~cm}$ : II $\mathrm{BC}_{1 \mathrm{~g}}$, idem mais bigarrures, marmorisation et dégradation hydromorphe subhorizontale mieux marquée, galets cristallins moins altérés ou moins intégrés à la matrice, limite inférieure graduelle, ondulée ;

$-220-380 \mathrm{~cm}:$ II $\mathrm{BC}_{2 \mathrm{~g}}$, idem mais fond matriciel moins vivement coloré, dominance des plages 5 Y $6 / 1$ à $5 \mathrm{Y} 6 / 2$, galets cristallins moins altérés, limite inférieure franche et ondulée sur la molasse ;

- $380-430 \mathrm{~cm}$ : II $\mathrm{BC}_{3 \mathrm{~g}}$, molasse marmorisée fond 10 YR $5 / 6$ à trainées verticales 5 Y $6 / 2$ et enduits 10 YR 3/1, structure massive.

Photo 13 - Terrasse du Mindel (arrêt $n^{\circ} 11$ ). Sol lessivé hydromorphe en surface, avec forte accumulation de concrétions (horizon noir). Epais profil d'altération argilisé, colmaté et bigarré. 


\begin{tabular}{|c|c|c|c|c|c|c|}
\hline \multirow{3}{*}{$\begin{array}{l}\text { Prof. } \\
\mathrm{cm}\end{array}$} & \multicolumn{6}{|c|}{ Granulométrie \% } \\
\hline & \multirow[b]{2}{*}{$\mathrm{EG}$} & \multicolumn{5}{|c|}{$\%$ de TF } \\
\hline & & SG & $\mathrm{SF}$ & STF & $\mathrm{L}$ & A \\
\hline $30-50$ & 46,4 & 6,5 & 17,7 & 27,6 & 20,7 & 26,1 \\
\hline $65-80$ & 72,5 & 11,9 & 13,0 & 16,6 & 14,2 & 43,4 \\
\hline $100-110$ & 79,0 & 11,9 & 12,1 & 13,3 & 12,4 & 48,8 \\
\hline $170-190$ & 57,8 & 16,0 & 14,2 & 10,5 & 14,8 & 43,5 \\
\hline $260-280$ & 25,8 & 31,6 & 12,8 & 7,8 & 12,9 & 33,9 \\
\hline $360-380$ & 57,5 & 46,9 & 15,9 & 7,3 & 10,4 & 19,0 \\
\hline $400-410$ & 0 & 1,9 & 15,6 & 14,9 & 20,3 & 46,8 \\
\hline
\end{tabular}

\begin{tabular}{|c|c|c|c|c|c|c|}
\hline \multirow{2}{*}{$\begin{array}{c}\text { Prof. } \\
\text { cm }\end{array}$} & eau & KCl & MO & C & \multicolumn{3}{c|}{ Matière organique \% } & C/N \\
\cline { 2 - 7 } & & & & & & \\
$30-50$ & 7,5 & 6,8 & 0,8 & 0,51 & 0,08 & 6,0 \\
$65-80$ & 6,7 & 5,1 & 0,3 & 0,18 & 0,05 & 3,2 \\
$100-110$ & 6,6 & 5,2 & 0,3 & 0,18 & 0,05 & 3,6 \\
$170-190$ & 5,8 & 4,0 & 0,2 & 0,14 & 0,03 & 4,0 \\
$260-280$ & 6,5 & 4,4 & 0,08 & 0,05 & 0,03 & 1,5 \\
$360-380$ & 7,3 & 5,8 & 0,01 & 0,01 & 0,02 & 0,4 \\
$400-410$ & 7,6 & 6,4 & 0,14 & 0,08 & 0,05 & 1,5 \\
\hline
\end{tabular}

\begin{tabular}{|c|c|c|c|c|c|c|c|}
\hline \multirow[b]{2}{*}{$\begin{array}{c}\text { Prof. } \\
\mathrm{cm}\end{array}$} & \multicolumn{7}{|c|}{ Complexe absorbant meq $\%$} \\
\hline & $\mathrm{Ca}$ & $\mathrm{K}$ & $\mathrm{Mg}$ & $\mathrm{Na}$ & $\mathrm{S}$ & $\mathrm{T}$ & $\mathrm{S} / \mathrm{T} \%$ \\
\hline $30-50$ & 11,1 & 0,27 & 0,85 & 0,06 & 12,3 & 13,7 & 89 \\
\hline $65-80$ & 12,9 & 0,32 & 3,5 & 0,06 & 16,8 & 23,7 & 70 \\
\hline $100-110$ & 15,3 & 0,38 & 4,7 & 0,16 & 20,7 & 26,7 & 77 \\
\hline $170-190$ & 17,4 & 0,32 & 6,0 & 0,32 & 24,2 & 25,0 & 96 \\
\hline $260-280$ & 14,6 & 0,23 & 4,8 & 0,57 & 20,2 & 25,0 & 80 \\
\hline $360-380$ & 11,9 & 0,12 & 3,1 & 0,54 & 15,7 & 19,3 & 81 \\
\hline $400-410$ & 19,7 & 0,32 & 6,1 & 0,76 & 27,0 & 27,7 & 97 \\
\hline
\end{tabular}

\begin{tabular}{|c|c|c|c|}
\hline \multirow[b]{2}{*}{$\begin{array}{l}\text { Prof. } \\
\mathrm{cm}\end{array}$} & \multicolumn{3}{|c|}{$\mathrm{Fe} \%$} \\
\hline & FT & $\mathrm{FL}$ & $\mathrm{FL} / \mathrm{FT} \%$ \\
\hline $30-50$ & 2,00 & 0,78 & 39 \\
\hline $65-80$ & 4,85 & 2,34 & 48 \\
\hline $100-110(a)$ & 4,95 & 2,90 & 58 \\
\hline $100-110(b)$ & 4,05 & 2,85 & 70 \\
\hline 120 & 0,90 & 0,56 & 62 \\
\hline $170-190(d)$ & 3,12 & 1,11 & 35 \\
\hline 180 & 3,40 & 1,00 & 30 \\
\hline 180 & 1,45 & 0,50 & 34 \\
\hline $260-280(\mathrm{~g})$ & 2,31 & 0,40 & 17 \\
\hline $360-380(h)$ & 1,84 & 0,40 & 22 \\
\hline $400-410$ (i) & 3,80 & 1,60 & 42 \\
\hline
\end{tabular}
(a) : échantillon total
(b) : plages les plus rouges
(c) : cristallin arénisé et argilisé rouille
(d) : matrice à dominante grise

(e) : cortex argileux gris d'un cristallin intégré

(f) : partie centrale d'un granite altéré gris

(g) et (h) : plages à dominante grise

(i) : molasse panachée 



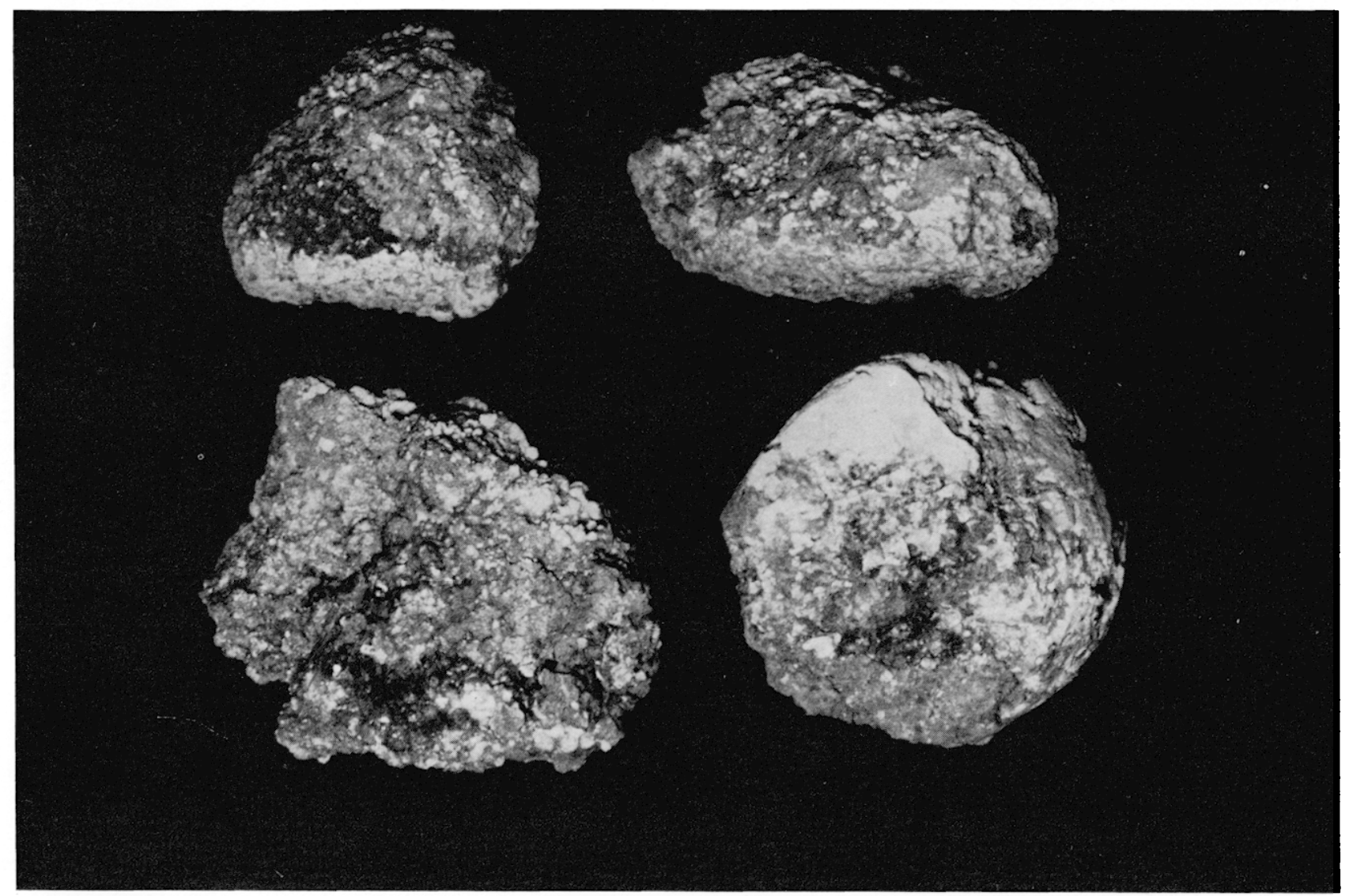

Photo 15 - Terrasse mindélienne Galets cristallins argilisés et plus ou moins intégrés à la matrice.

\begin{tabular}{|c|c|c|c|c|}
\hline $\begin{array}{l}\text { Prof. } \\
\mathrm{cm}\end{array}$ & Illites & $\mathrm{C}-\mathrm{V}-\mathrm{Al}^{*}$ & Kaolinites & $\begin{array}{l}\text { Interstr. } \\
\text { gonflants }\end{array}$ \\
\hline $\begin{array}{c}30-50 \\
65-80 \\
100-110 \\
170-190 \\
260-280 \\
360-380 \\
400-410 \\
\text { Galet cristallin } \\
\text { altéré et argilisé, } \\
\text { vers } 150 \mathrm{~cm} ; \\
\text { Cortex } \\
\text { Centre }\end{array}$ & $\begin{array}{l}\mathrm{M} \\
\mathrm{M} \\
\mathrm{F} \\
\mathrm{F} \\
\mathrm{M} \\
\mathrm{F} \\
\mathrm{F}\end{array}$ & $\begin{array}{l}\mathrm{F} \\
\mathrm{M} \\
\mathrm{m} \\
\mathrm{f} \\
\mathrm{f} \\
\mathrm{M} \\
\mathrm{M}\end{array}$ & $\begin{array}{l}\mathrm{f} \\
\mathrm{m} \\
\mathrm{M} \\
\mathrm{M} \\
\mathrm{m} \\
\mathrm{tr}\end{array}$ & $\begin{array}{c}\text { tr. } \\
\text { f } \\
\text { f } \\
\text { f } \\
\text { tr. } \\
\text { m } \\
\\
\text { f } \\
\text { présence } \\
\text { de } \\
\text { gibbsite }\end{array}$ \\
\hline
\end{tabular}

(*) C-V-Al : minéraux à comportement de vermiculite ou chlorite-Al, ou d'interstratifiés 10-14 C, 10-14 V, 14 C-14 V.

- Le gradient vertical d'altération dans les alluvions caillouteuses apparaît ici dans toute son ampleur, malgré l'épaisseur relativement restreinte de la nappe. Il concerne d'abord la séquence progressive d'enrichissement (avec la profondeur) en éléments caillouteux cristallins de moins en moins altérés (surtout granites et gneiss). De fait, tout au sommet du cailloutis, un très grand nombre de ces éléments se trouvent soit réduits à l'état de "fantômes" inclus dans la matrice, soit, plus ou moins pulvérulents et argilisés, en voie d'intégration à la matrice (photos 14 et 15); les galets relativement peu altérés sont exceptionnels. Ces derniers deviennent en revanche de moins en moins rares avec la profondeur et, de façon générale, le nombre des éléments cristallins encore plus ou moins cohérents, quoique fissurés et fragiles, tend à augmenter. $\mathrm{Au}$ contact de la molasse, ce schéma est parfois brouillé en raison de l'altération provoquée par l'hydromorphie 
"remontante" de la nappe d'eau perchée supramolassique.

- L'illuviation et l'argilisation dans le cailloutis ressortissent à la séquence progressive d'appauvrissement de la matrice, évoquée dans les pages précédentes. Sous le profil différencié dans les limons caillouteux (ici exceptionnellement chargés d'éléments grossiers abondants), les taux d'argiles culminent vers le sommet de la grave, dans le II $B_{3 \text { tg }}$, et diminuent d'abord lentement puis un peu plus rapidement vers le bas, pour remonter très nettement ensuite dans le III $\mathrm{BC}_{3 \mathrm{~g}}$ molassique. Il $\mathrm{y}$ a donc à la fois estompage de l'illuviation verticale et décroissante de l'argilogénèse in situ (1). On notera l'importance de ces taux d'argile, même sous l'horizon franchement argillique II $B_{3 \text { tg }}$.

(1) Cette argilogénèse in situ nous semble avoir joué un rôle important dans l'argilisation du cailloutis, contrairement à l'interprétation suggérée par $\mathbf{N}$. Fédoroff, selon laquelle le nourrissement des graves traduirait essentiellement l'illuviation. Certains faits d'observation contredisent ce point de vue, par exemple le faible taux d'argile de bancs locaux de sables

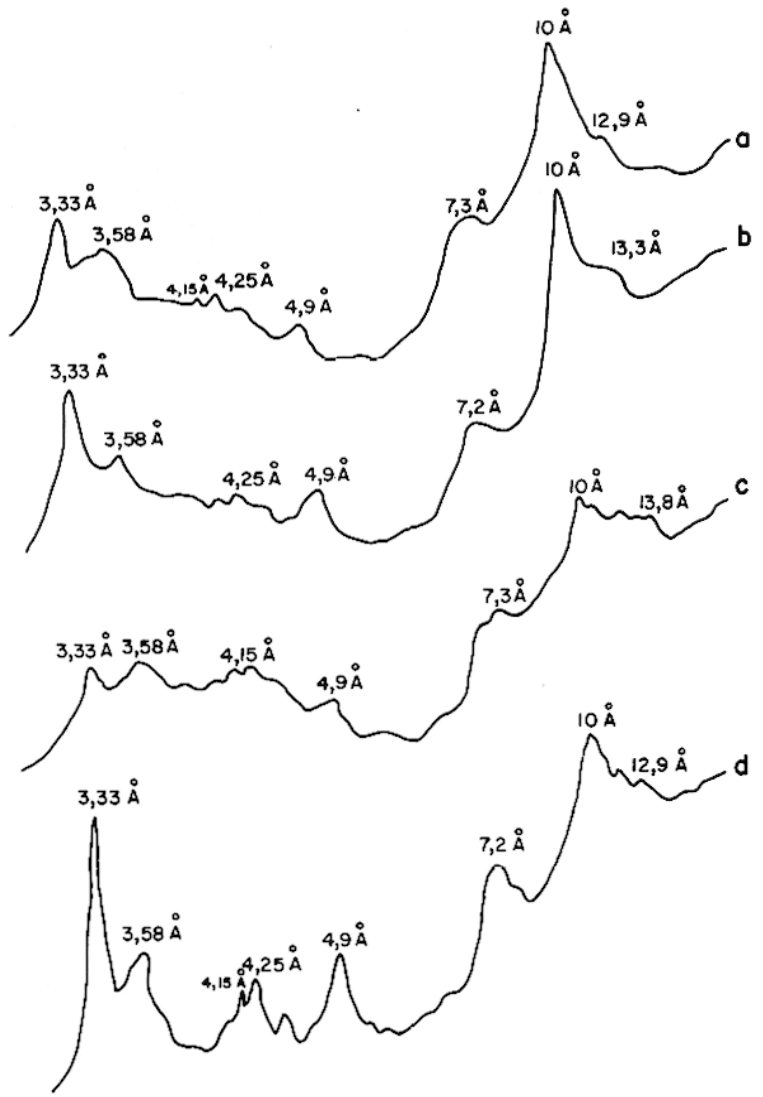

Comparés aux profils rissiens et würmiens, il s'agit là d'un caractère tout à fait spécifique des remblaiements prérissiens.

- L'ambiance ionique qui baigne le cailloutis mindélien diffère également notablement de celle des matériaux rissiens et würmiens. D'une part, les $\mathrm{pH}$ sont généralement plus faibles, ce que le profil de l'arrêt $n^{\circ} 11$, perturbé par un fort marnage molassique en surface, montre malheureusement très mal. D'autre part, la matrice du cailloutis constitue une véritable mosaïque de $\mathrm{pH}$, lesquels oscillent point par point entre moins de 5 et plus de 7 , caractère qui tranche avec la belle régularité des profils rissiens et würmiens. Cette propriété, que l'on retrouvera dans le Günz, traduit à la fois le degré variable d'altération des

grossiers quartzeux, inclus dans les horizons II BC. En outre, on s'expliquerait mal, dans cette hypothèse, la variation verticale du cortège de minéraux argileux (cf. pages suivantes). Sans nier la part d'illuviation, on a donc choisi de symboliser ces horizons profonds par des II BC plutôt que par des II $B_{t} C$.

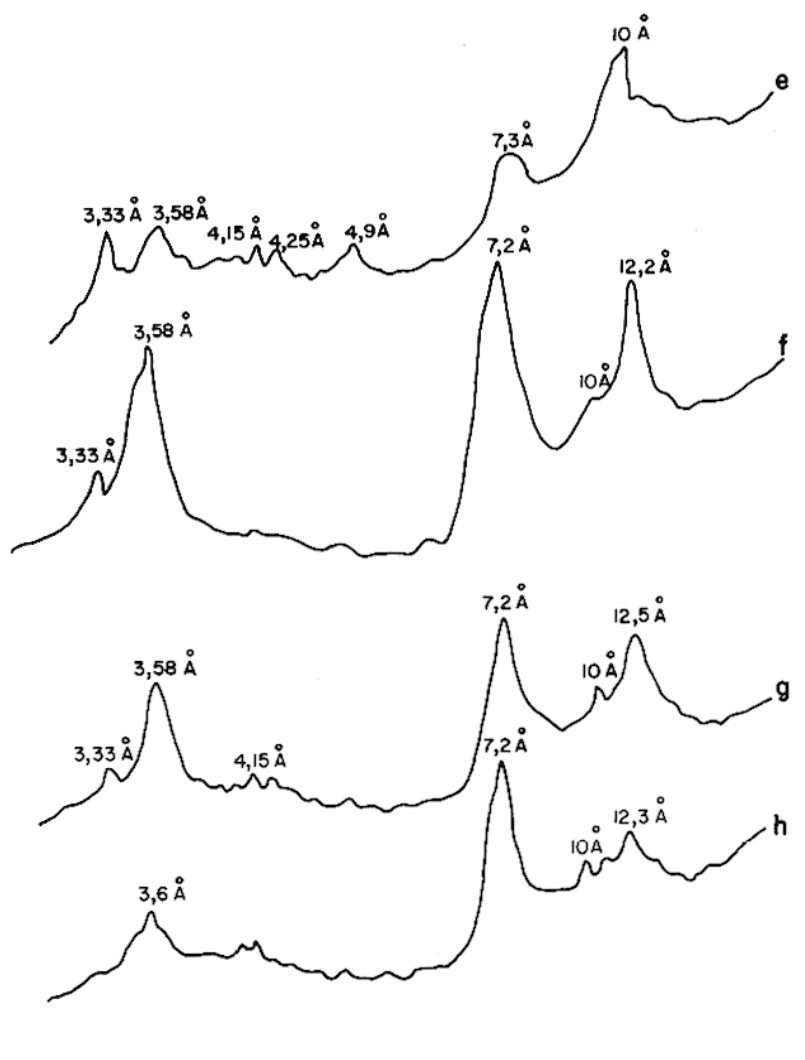

Fig. 14 - Abondance et cristallinité différentielle des kaolinites dans les matrices des cailloutis prérissiens (horizons sommitaux). a*, b, c : Mindel ; $\mathrm{d}^{*}$, e : Günz; $\mathrm{f}^{*}, \mathrm{~g}, \mathrm{~h}$ : Donau.

$a^{*}$ : coupe de l'arrêt $n^{\circ} 11,170-190 \mathrm{~cm}$

$\mathrm{d}^{*}$ : coupe de l'arrêt $\mathrm{n}^{\circ} 12,160-170 \mathrm{~cm}$

$f^{*}$ : coupe d'Escazeaux, $150-160 \mathrm{~cm}$

Dans le Mindel et le Günz, les minéraux kaoliniques sont relativement abondants (raies à 7,2 - 7,3 $\AA$ et 3,58 $\AA$ ), mais généralement subordonnés au groupe formé par les illites et les minéraux à $10-14 \AA$. Leurs raies, notamment celles à $7,2-7,3 \AA$, sont larges, émoussées, très dissymétriques et traduisent le caractère désordonné des structures cristallines. Toutefois, dans le Günz, elles offrent la plupart du temps un degré d'organisation moins médiocre que dans les terrasses moyennes. Dans la nappe du Donau en revanche, les kaolinites, qui constituent une large partie du stock d'argiles, apparaissent bien cristallisées : pics nets à 7,2 $\AA$ et 3,58 - $3,60 \AA ;$ bien dégagés du "bruit de fond", aigus et beaucoup moins dissymétriques. On note, dans toutes ces nappes argilisées, la présente fréquente de goethite (raie modeste à $4,15 \AA$ ), hydroxyde qui s'observe cependant aussi dans les graves des basses terrasses de l'avant-pays. 
éléments rocheux et des minéraux primaires intégrés à la matrice, ainsi que la diversité quasi-ponctuelle des conditions de drainage à l'intérieur du cailloutis colmaté.

- La dynamique du fer reflète avant toute chose les processus liés à l'hydromorphie. Le sol lessivé hydromorphe développé dans la couverture de limons caillouteux montre, en profondeur, un net horizon à concrétions et enduits ferro-manganiques qui revêt par endroit un discret faciès de grep tendre (au contact de la grave).

Le cailloutis alluvial, intensément bariolé, trahit l'intense redistribution du fer liée à l'altération différentielle des minéraux primaires et à l'important colmatage de la grave. Apparemment anarchique dans le détail (imbrication complexe des plages claires et colorées), la redistribution du fer s'organise beaucoup plus clairement à un niveau supérieur, notamment sous forme de "bandes de dégradation" grises ou beige très clair, généralement subhorizontales et particulièrement bien exprimées à la base des horizons II $\mathbf{B}_{\mathrm{tg}}$. Les cortex gris ou rouille développés à la surface des galets (surtout des quartzites) traduisent essentiellement l'environnement matriciel immédiat, gris ou coloré, de ces éléments caillouteux (cf. arrêt $\mathrm{n}^{\circ} 12$ ).

De cette intense réorganisation du fer, il résulte que la séquence progressive d'appauvrissement en fer de la matrice, précédemment évoquée, se trouve totalement perturbée : plages déferrifiées et plages enrichies en fer se juxtaposent tout le long du profil, et masquent radicalement le gradient vertical de redistribution du fer (cf. tableau d'analyse).

De la même façon et pour les mêmes raisons, la rubéfaction de la matrice ne manifeste pas très clairement: le phénomène est considérablement brouillé par l'hydromorphie et la redistribution du fer qu'elle provoque. Cependant, là où la réorganisation liée à l'hydromorphie n'est pas très intense, la rubéfaction de la matrice est plus nette que dans les profils rissiens : ainsi, au sommet du cailloutis, l'horizon II $B_{3 \text { tg }}$ montre de larges plages colorées à 5 YR $5 / 8$ à $5 / 6$. La goethite s'y révèle dans les diffractogrammes (comme du reste dans toutes les plages colorées de l'alluvion caillouteuse, et également assez souvent au. dessus des fronts d'altération du Riss); l'hématite n'a pu y être observée, sinon tout à fait exceptionnellement.

On notera, en dernier lieu, le faciès de réorganisation hydromorphe de la molasse sous-jacente. Les larges traînées verticales, grises et gainées de rouille, évoquent de façon saisissante les glosses des couvertures limoneuses antérissiennes (cf. arrêt $\mathrm{n}^{\circ} 15$ ).

- L'évolution des minéraux argileux, dans le profil superficiel limono-caillouteux, s'apparente étroitement à celle des couvertures limoneuses rissiennes. La ma- trice des cailloutis, en revanche, se singularise radicalement, en raison de l'émergence de structures kaoliniques déjà relativement abondantes. Mais ces kaolinites apparaissent, en diffractométrie, comme très désordonnées et encore très médiocrement cristallisées (fig. $14 \mathrm{a}, \mathrm{b}, \mathrm{c}$ ) et s'apparentent alors plus ou moins à des métahalloysites. D'autre part, ces minéraux deviennent assez rapidement de moins en moins abondants avec la profondeur; parallèlement à cette décroissance, le degré de désorganisation et la médiocre cristallinité des kaolinites s'accentuent sensiblement, comme le suggère la mauvaise définition des diffractogrammes $X$. Enfin, on notera la présence de gibbsite au centre de certains galets cristallins altérés.

Ainsi, aux séquences définies précédemment s'ajoute ici une nouvelle séquence progressive verticale, liée au gradient vertical d'altération et portant à la fois sur la teneur en minéraux kaoliniques et sur leur degré d'organisation.

- L'attribution au Mindel des alluvions de la terrasse moyenne se justifie par le fait qu'il s'agit de la première nappe très franchement altérée, argilisée, colmatée et plus ou moins rubéfiée, au-dessus de la basse terrasse rissienne, encore relativement peu affectée par tous ces phénomènes. L'outillage, de tradition acheuléenne, fréquemment récolté à la surface de la terrasse moyenne (H. Breuil 1937, A. Cavaillé 1969b), est sans doute nettement postérieur à la mise en place du remblaiement alluvial, ce que les observations de A. Tavoso (1975) confirment pour la région tarnaise.

\section{II. - LA TERRASSE GUNZIENNE ET LES CUIRASSEMENTS FERRUGINEUX ASSOCIES AUX ALLUVIONS}

\section{1 - Organisation pédo-géochimique des remblaiements güziens}

Nettement plus démantelé que la terrasse mindélienne, le niveau günzien se présente, dans la Forêt de Bouconne, sous la forme d'un plateau de $230-240 \mathrm{~m}$ d'altitude, qui constitue la partie médiane de la forêt domaniale. Ce plateau est entaillé de quelques vallons et c'est précisément à proximité immédiate de l'un d'entre eux que se trouve la coupe de l'arrêt $n^{\circ} 12$ (photo 16). Ici, le remblaiement alluvial atteint environ 6 à $7 \mathrm{~m}$ au-dessus de la molasse, mais le front de coupe ne révèle que 4 à $4,5 \mathrm{~m}$ de matériel.

\section{Description sommaire du profil de l'arrêt $n^{\circ} 12$ :}

Sol lessivé hydromorphe, profil d'altération à tendance fersiallitique

- 0-30 cm: Ap. 10 YR 6/3, structure massive, sous-structure polyédrique plate, peu cohérent. racines, 
pores, graviers et galets TRA $10 \%$, limite inférieure graduelle, ondulée ;

$-30-55 \mathrm{~cm}: A_{2 g}$ et $B_{1 t g}$, fond 10 YR 6/4 à taches rouille, structure massive à̀ tendance polyédrique, racines, pores, graviers et gravillons TRA 5 à $10 \%$, quelques revêtements argileux, enduits et concrétions noirs de fer surtout au contact de la grave, limite inférieure franche et ondulée (poches);

- 55-210 cm: II $B_{2 t g}$ puis IIBC $_{1 \mathrm{~g}}$, nappe caillouteuse TRA 40 à $60 \%$ puis 60 à $70 \%$, matrice fond 7,5 YR 6/6 à 5 YR 4/8, avec bandes subhorizontales et verticales 10 YR $7 / 2$ à $7 / 3$ et plages d'enduits ou concrétions noires, épais revêtements argileux, cortex gris ou rouille des quartzites, rares galets cristallins type fantômes ou très intégrés à la matrice, limite inférieure graduelle et indistincte ;
$-210-270 \mathrm{~cm}: \mathrm{IIBC}_{2 \mathrm{~g}}$, idem mais matrice fond 7 YR $5 / 8$ à 10 YR $5 / 8$ à $5 / 3$, avec larges plages $2,5 \mathrm{Y}$ $6 / 2$ à 5 Y $5 / 3$ et zones noires TRA 10 à $20 \%$ cristallins très altérés moins rares, limite inférieure graduelle, indistincte ;

- 270-320 cm: IIBC $_{3 \mathrm{~g}}$, même type d'organisation mais ensemble matriciel très bigarré, galets cristallins déjà plus abondants quoique altérés et souvent intégrés à la matrice, parfois encore légèrement cohérents, limite inférieure graduelle, indistincte ;

$-320-450 \mathrm{~cm}$ : $\mathrm{IlBC}_{4 \mathrm{~g}}$, idem mais éléments cristallins nettement plus nombreux, parfois encore assez cohérents, revêtements argileux plus rares, ensemble matriciel toujours très bigarré.

\begin{tabular}{|c|c|c|c|c|c|c|}
\hline \multirow{2}{*}{$\begin{array}{c}\text { Prof. } \\
\text { cm }\end{array}$} & \multirow{6}{|c|}{ Granulométrie \% } \\
\cline { 2 - 7 } & EG & SG & SF & STF & L & A \\
\cline { 2 - 7 } & & 3,6 & 13,4 & 33,5 & 22,1 & 23,4 \\
$15-25$ & 11,5 & 3,6 & 11,5 & 32,9 & 22,2 & 27,9 \\
$40-50$ & 12,9 & 4,7 & 9,7 & 23,5 & 18,0 & 42,3 \\
$55-80$ & 44,7 & 8,2 & 9,5 & 12,7 & 12,4 & 55,5 \\
$160-170$ & 52,1 & 12,6 & 16,1 & 11,4 & 14,0 & 44,1 \\
$240-250$ & 41,0 & 23,3 & 19,1 & 8,6 & 13,6 & 34,4 \\
$280-300$ & 63,6 & 17,2 & 18,4 & 11,2 & 15,6 & 36,6 \\
$340-360$ & 67,5 & & & & & \\
\hline
\end{tabular}

\begin{tabular}{|c|c|c|c|c|c|c|}
\hline \multirow[b]{2}{*}{$\begin{array}{c}\text { Prof. } \\
\mathrm{cm}\end{array}$} & \multicolumn{2}{|c|}{$\mathrm{pH}$} & \multicolumn{4}{|c|}{ Matière organique \% } \\
\hline & eau & $\mathrm{KCl}$ & MO & $\mathrm{C}$ & $\mathrm{N}$ & $\mathrm{C} / \mathrm{N}$ \\
\hline $15-25$ & 4,5 & 3,9 & 1,5 & 0,91 & 0,06 & 14,0 \\
\hline $40-50$ & 4,7 & 3,9 & 1,1 & 0,66 & 0,05 & 11,8 \\
\hline $55-80$ & 4,3 & 3,7 & 0,8 & 0,51 & 0,05 & 9,1 \\
\hline $160-170$ & 4,7 & 3,5 & 0,7 & 0,44 & 0,04 & 9,0 \\
\hline $240-250$ & 5,9 & 4,4 & 0,9 & 0,54 & 0,04 & 13,2 \\
\hline $280-300$ & 7,0 & 5,8 & 0,4 & 0,28 & 0,03 & 9,1 \\
\hline $340-360$ & 7,5 & 6,5 & 0,5 & 0,34 & 0,03 & 9,6 \\
\hline
\end{tabular}

\begin{tabular}{|c|c|l|l|l|l|l|l|l|l|l|l|}
\hline & \multicolumn{9}{|c|}{ Complexe absorbant meq \% } & \multicolumn{3}{c|}{ Fe \% } \\
\cline { 2 - 10 } $\begin{array}{c}\text { Prof. } \\
\mathrm{cm}\end{array}$ & $\mathrm{Ca}$ & $\mathrm{K}$ & $\mathrm{Mg}$ & $\mathrm{Na}$ & $\mathrm{S}$ & $\mathrm{T}$ & $\mathrm{S} / \mathrm{T} \%$ & $\mathrm{FT}$ & $\mathrm{FL}$ & $\mathrm{FL} / \mathrm{FT} \%$ \\
\hline $15-25$ & 0,21 & 0,1 & 0,15 & 0,03 & 0,5 & 8,4 & 5 & 1,84 & 0,78 & 42 \\
$40-50$ & 0,35 & 0,12 & 0,75 & 0,03 & 1,3 & 8,9 & 14 & 2,28 & 1,28 & 56 \\
$55-80$ & 0,35 & 0,23 & 2,8 & 0,06 & 3,4 & 14,8 & 22 & 3,32 & 1,78 & $53(\mathrm{~b})$ \\
$70-75$ & & & & & & & & 3,01 & 1,22 & $40(\mathrm{a})$ \\
$160-170$ & 4,7 & 0,21 & 4,9 & 0,19 & 10,1 & 21,9 & 45 & 4,85 & 3,18 & $65(\mathrm{~b})$ \\
$240-250$ & 14,6 & 0,21 & 5,0 & 0,60 & 20,4 & 23,0 & 88 & 3,85 & 2,65 & $68(\mathrm{~b})$ \\
$280-300$ & 14,4 & 0,14 & 3,9 & 0,73 & 19,2 & 21,0 & 91 & 3,45 & 2,51 & $72(\mathrm{~b})$ \\
$340-360$ & 15,1 & 0,21 & 4,0 & 0,76 & 20,1 & 20,9 & 96 & 3,40 & 3,07 & $90(\mathrm{~b})$ \\
\hline
\end{tabular}

(a) Bande grise

(b) Echantillon global ou à dominante rubéfiée. 


\begin{tabular}{|c|c|c|c|c|}
\hline $\begin{array}{l}\text { Prof. } \\
\mathrm{cm}\end{array}$ & Illites & $\mathrm{C}-\mathrm{V}-\mathrm{Al}$ & Kaolinites & $\begin{array}{c}\text { Interstratifiés } \\
\text { gonflants }\end{array}$ \\
\hline $\begin{array}{c}15-25 \\
40-50 \\
160-170 \\
240-250 \\
280-300 \\
340-360 \\
410-430 \\
\text { Fantôme } \\
\text { de } \\
\text { cristallin } \\
\text { vers } 270 \mathrm{~cm} \\
\text { cortex. . } \\
\text { cœur. . } \\
\text { Cristallin } \\
\text { altéré } \\
\text { vers } 430 \mathrm{~cm} \\
\text { cortex. . } \\
\text { cœur. . }\end{array}$ & $\begin{array}{l}\mathrm{m} \\
\mathrm{M} \\
\mathrm{M} \\
\mathrm{M} \\
\mathrm{F} \\
\mathrm{F} \\
\mathrm{F}\end{array}$ & $\begin{array}{c}\mathrm{F} \\
\mathrm{F} \\
\mathrm{m} \\
\mathrm{f} \\
\mathrm{f} \\
\mathrm{M} \\
\mathrm{m}\end{array}$ & $\begin{array}{l}\mathrm{tr} \\
\mathrm{M} \\
\mathrm{F} \\
\mathrm{M} \\
\mathrm{m} \\
\mathrm{m}\end{array}$ & $\begin{array}{c}\text { f } \\
\\
\text { f } \\
\text { présence de } \\
\text { gibbsite }\end{array}$ \\
\hline
\end{tabular}

- L'organisation générale du profil, dans la nappe günzienne, reste assez proche de celle qui s'observe dans le Mindel. On retrouve ici le même type de séquences progressives que précédemment. Le mode de différenciation pédologique de la couverture limoneuse n'offre pas, lui non plus, de caractères fondamentalement nouveaux. On notera cependant que le manteau limoneux de l'arrêt $\mathrm{n}^{\circ} 12$ est très remanié et mis en place, pour une large part, par le ruissellement ou la solifluxion (bordure de vallon). Le profil de l'arrêt $\mathrm{n}^{\circ} 13$, situé au pied de versant d'un autre vallon, permet d'étudier plus précisément la différenciation en sols lessivés hydromorphes de ces limons remaniés.

- Plus que par la nature, c'est par le degré de réorganisation des profils que le Günz se distingue du Mindel.

- La séquence progressive d'appauvrissement de la matrice en argiles d'illuviation et d'altération in situ, se marque sur une plus grande profondeur du profil. Les horizons $\mathrm{UB}_{\mathrm{t}}$, franchement argilliques, sont généralement un peu plus épais. En outre, l'illuviation et surtout l'argilogénèse demeurent importantes dans les horizons les plus profonds, comme le montrent les taux d'argile encore élevés (quelques sondages, effectués à la tarière vers $5-6 \mathrm{~m}$ de profondeur à l'arrêt $\mathrm{n}^{\circ} 12$, donnent entre 25 et $30 \%$ d'argile).

- La séquence progressive d'enrichissement en éléments caillouteux cristallins de moins en moins altérés (granites et gneiss surtout), s'allonge de la même façon. Le sommet du cailloutis ne renferme plus que très rarement des galets cristallins reconnaissables: la plupart d'entre eux se trouvent réduits à l'état de fantômes ou totalement intégrés au matériau matriciel. Peu à peu, cependant, dès le bas des horzons $\mathrm{IIB}_{t}$ puis dans les horizons sous-jacents, la proportion de cailloux cristallins reconnaissables et parfois encore plus ou

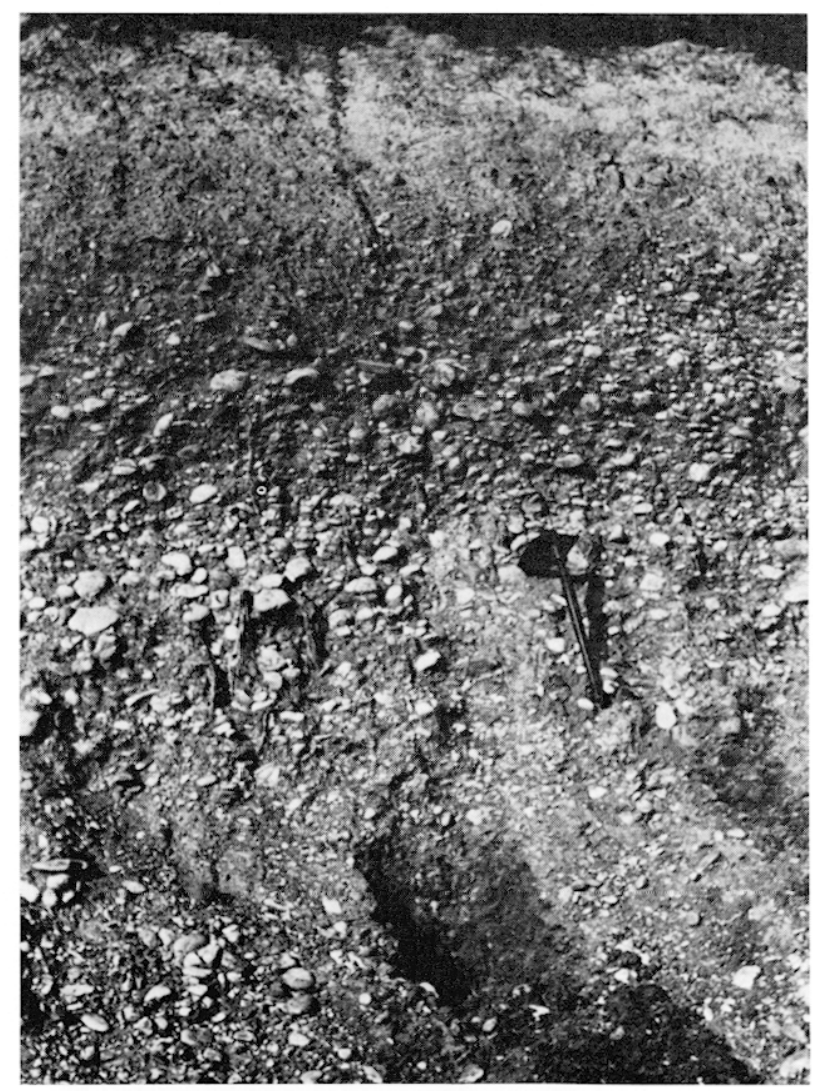

Photo 16 - Terrasse du Günz. Sol lessivé hydromorphe et cailloutis argilisé, colmaté, bigarré, plus coloré au sommet (teinte sombre).

moins cohérents, s'élève dans les spectres pétrographiques (figure 15). Vers $6 \mathrm{~m}$ de profondeur, on retrouve un stock de galets cristallins altérés qui reflète à peu près la proportion originelle de ces éléments dans le cailloutis alluvail frais (proportion de l'ordre de 30 à $50 \%$ ). 


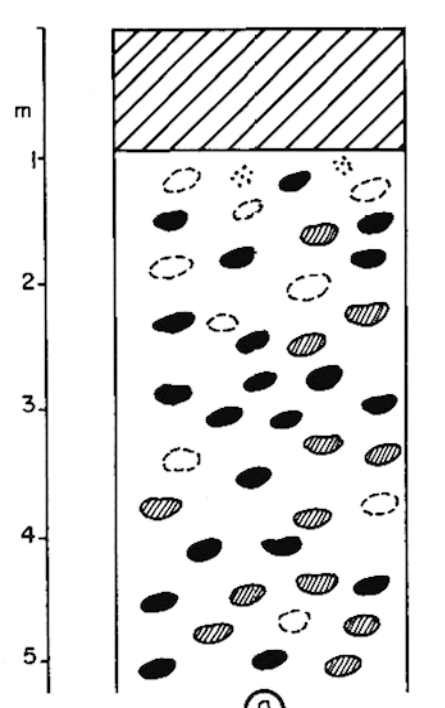

(a)
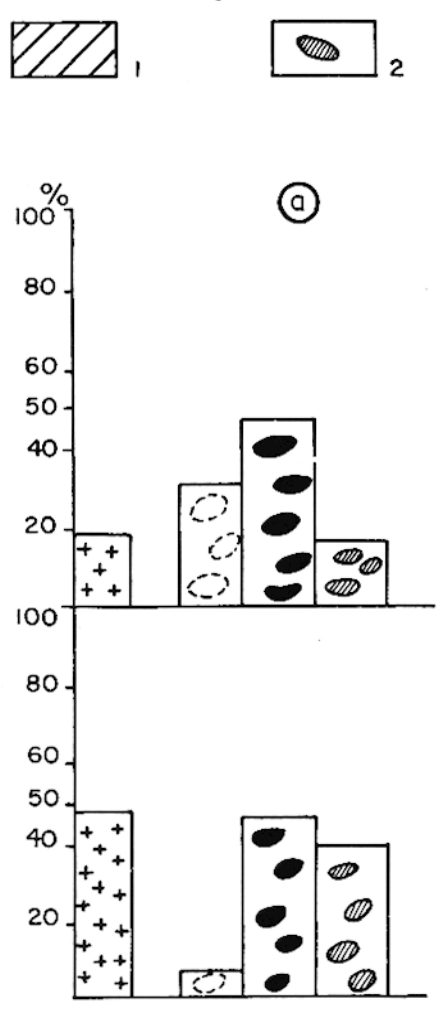

A
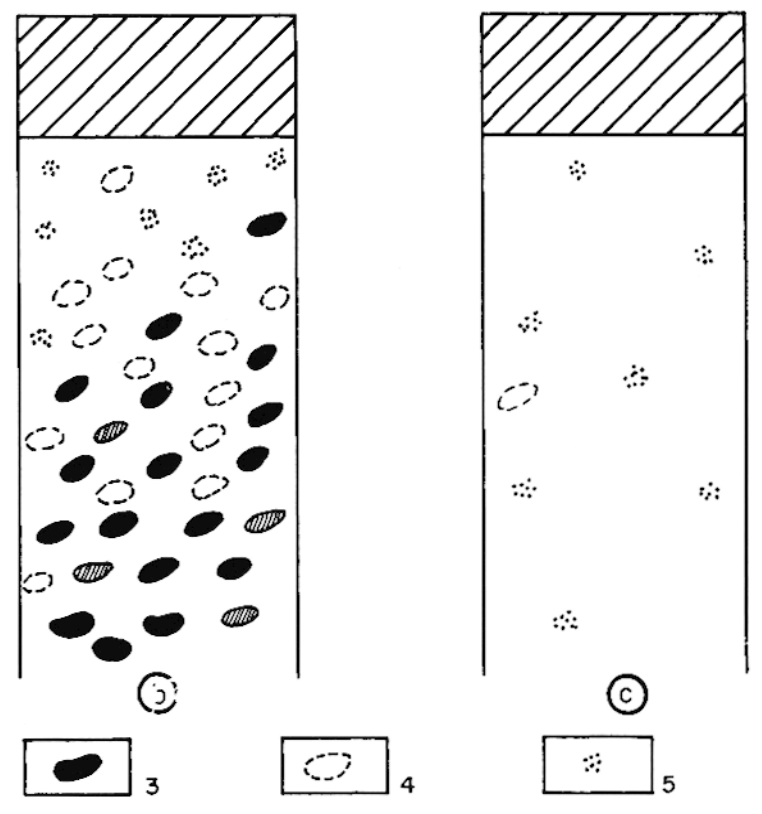

B

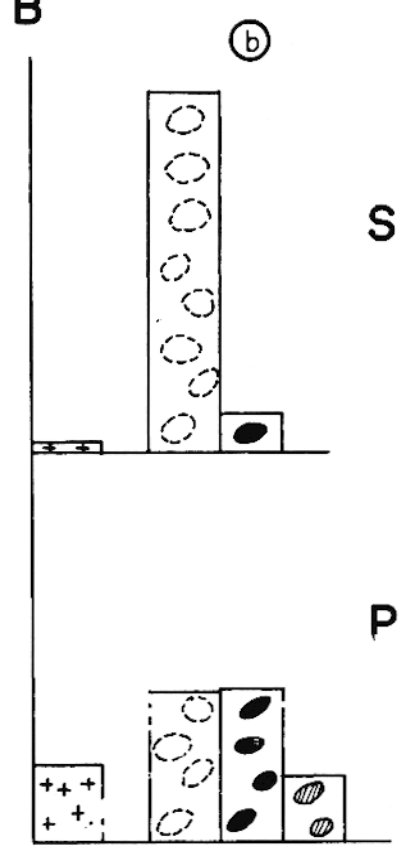

Fig. 15 - Gradient d'altération des galets de roches cristallines grenues dans les profils des nappes graveletses argilisées et colmatées. A. Gradient d'altération des galets cristallins avec la profondeur. 1. Couverture limoneuse 2. Galet cristallin altéré, peu intégré à la matrice intersticielle 3. Galet cristallin fortement altéré, en voie d'intégration marquée à la matrice 4 . Résidus de galets cristallins à faciès de "fantôme" 5. Amas blanchâtres non identifiables, résultant de la désintégration totale des galets cristallins.

a. Terrasse Mindel b. Terrasse Günz c. Nappe culminante Donau.

B. Proportions de roches cristallines grenues de différents faciès d'altération. 6. Proportion globale d'éléments cristallins encore identifiables, rapportée au nombre total de galets de toute nature S. Sommet des nappes graveleuses $\mathbf{P}$. Horizons profonds des nappes graveleuses.

. La dynamique et la redistribution du fer dans la nappe günzienne ne diffèrent pas sensiblement de celles qui marquent les profils mindéliens. Tout au plus l'empreinte de l'hydromorphie est-elle mieux exprimée ici : bandes subhorizontales et obliques grises de "dégradation" plus continues et plus apparentes ; présence plus fréquente et caractère plus affirmé des cortex gris ou rouille développés à la surface des galets de quartzites (photo 17), en rapport avec la meilleure expression de la redistribution du fer (fig. 16).

De la même façon que dans le Mindel, cette intense redistribution du fer brouille considérablement la sé- 


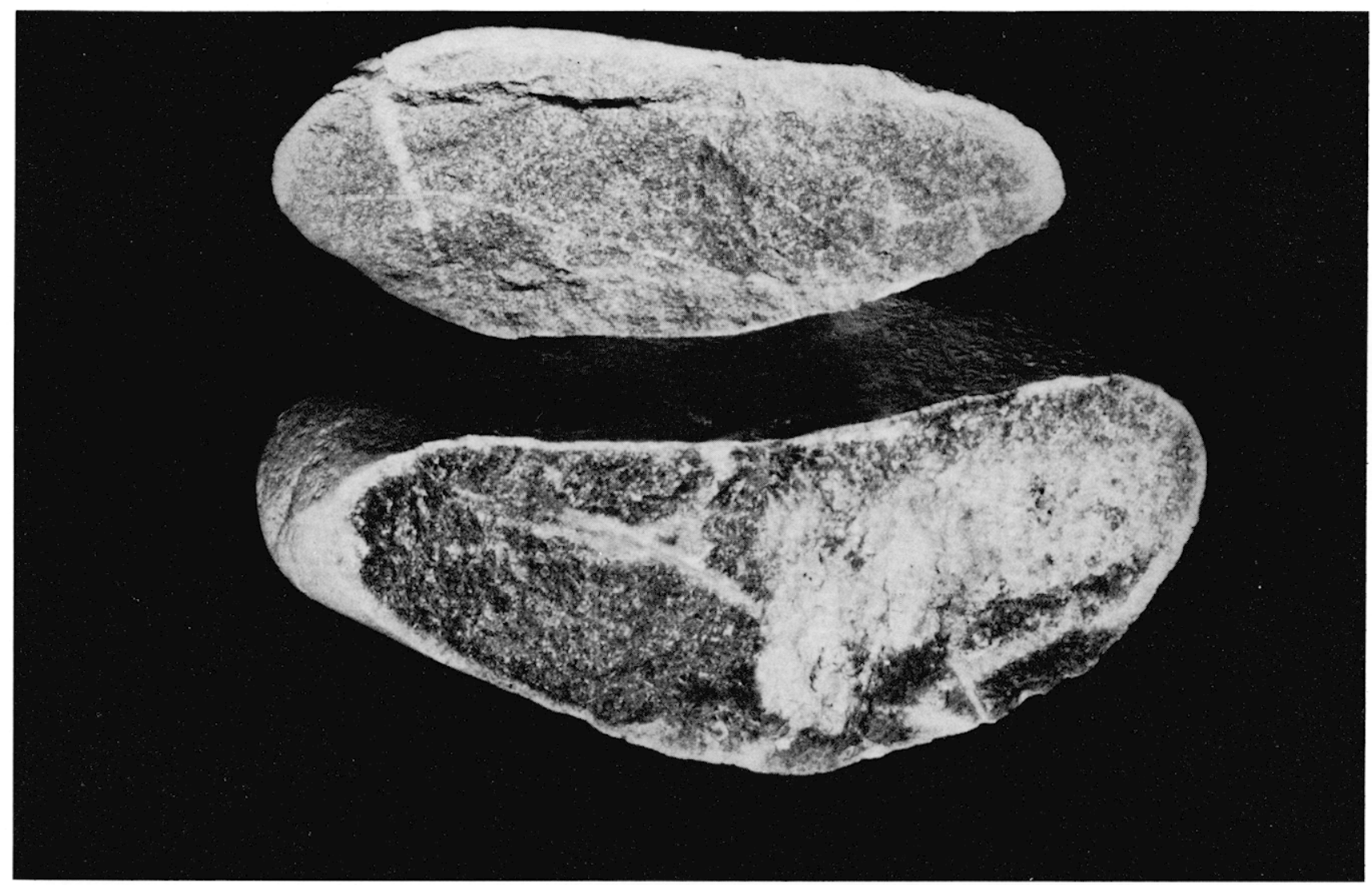

Photo 17 - Terrasse günzienne. Allure des cortex de quartzites. Cortex blanc-gris plus ou moins épais, interrompus par de minces cortex ou liserés rouille. Présence locale de cortex ou liserés rouille internes, sous le cortex gris.

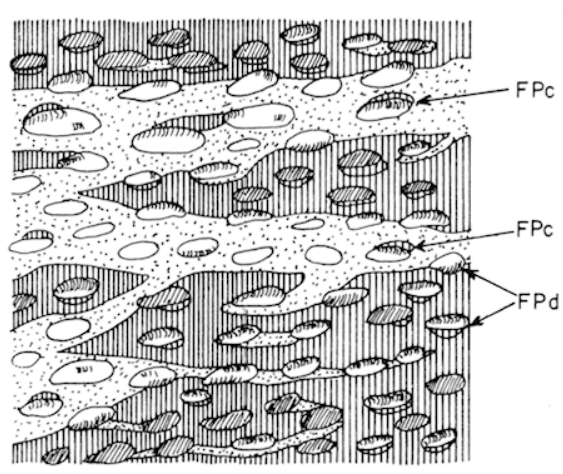

a

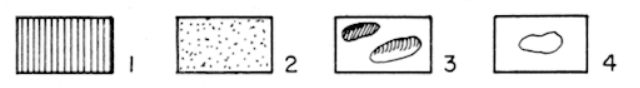

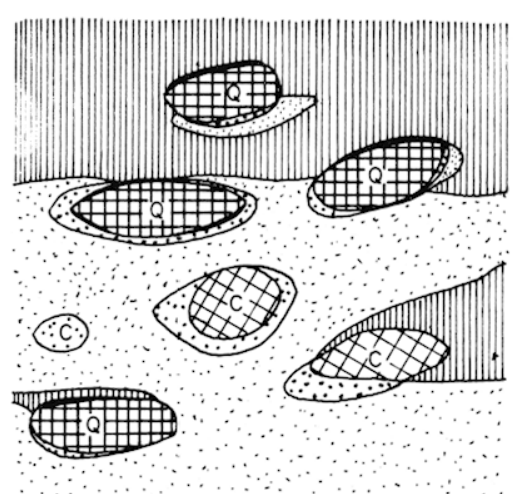

$\mathrm{b}$

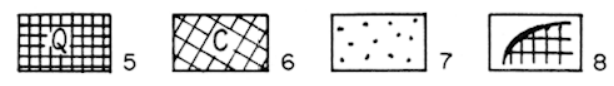

Fig. 16 - Esquisse schématique des faciès de redistribution du fer dans les nappes graveleuses argilisées et colmatées.

1. Matrice intersticielle riche en fer, colorée 2. Matrice intersticielle déferrifiée, grise (bandes, langues et faces de pression) 3 . Galets entièrement ou partiellement patinés en brun-rouge ou rouille 4 . Galets à surface claire 5 . Cœur dur et frais des quartzites 6 . Cœur altéré généralement rouille ou jaunâtre clair des galets cristallins 7 . Zones corticales grises 8 . Liseré ou patine rouille ou brun-rouge des quartzites F Pc. Faces de pression argileuses des galets, brun-rouge ou rouille F Pd. Faces de pression argileuses des galets, grises.

quence verticale ferrugineuse de la matrice (cf. tableau d'analyses) et provoque, une fois encore, la confusion entre faciès rubéfiés et faciès enrichis en fer de redistribution hydromorphe. Toutefois, à l'arrêt $\mathrm{n}^{\circ} 12$, la tranche supérieure du cailloutis est, dans son ensemble, un peu plus vivement colorée que les horizons homologues du profil mindélien de l'arrêt $\mathrm{n}^{\circ} 11$ : ce fait est à mettre en relation avec le site de drainage et le pédoclimat qui en résulte. 


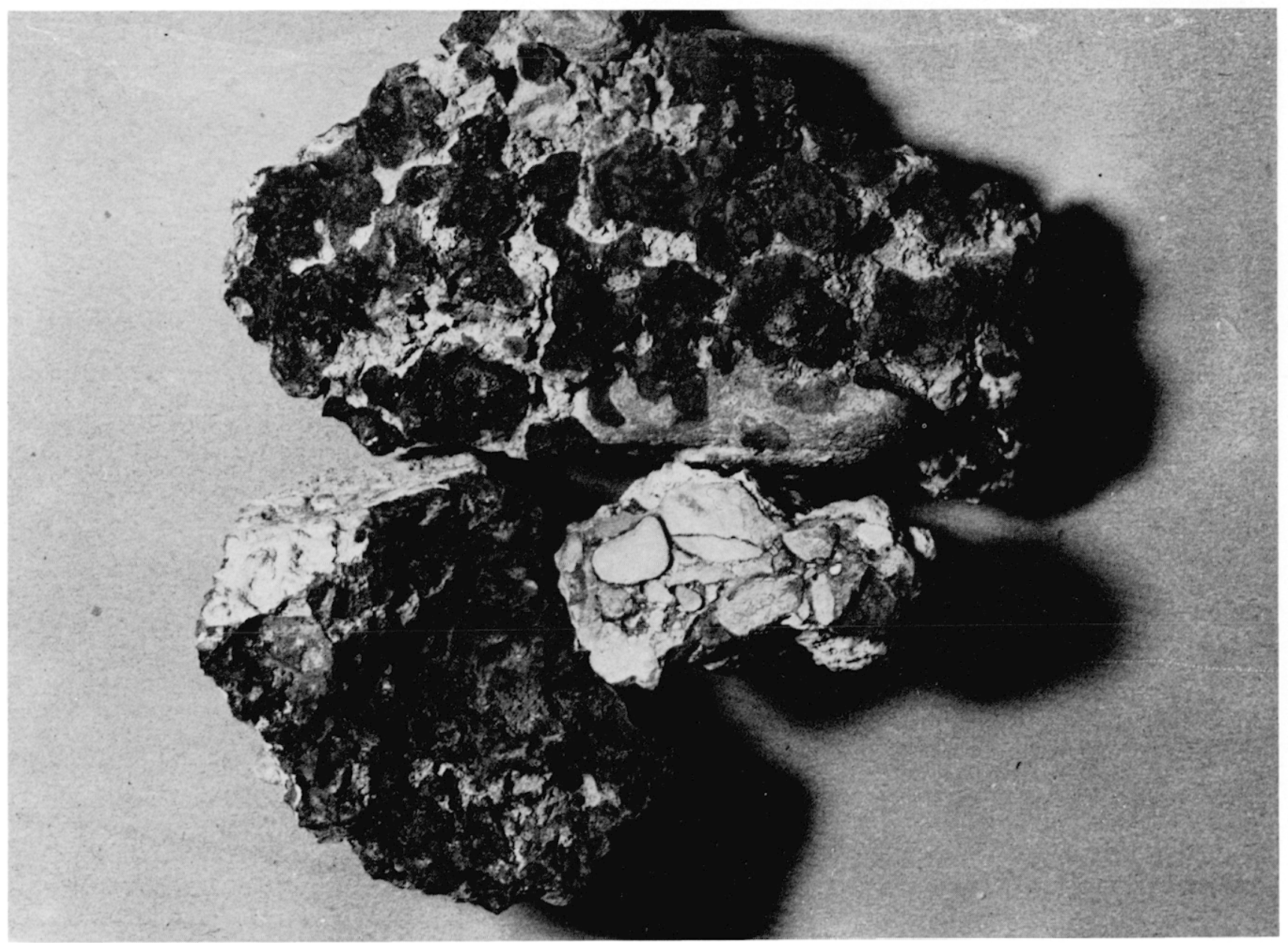

Photo 18 - Cuirassements ferrugineux (greps). En haut, élément de grep dur, dont une coupe type "nougat" est donnée au centre. En bas, élément de noyau cuirassé, d'allure scoriacée.

- La séquence progressive portant sur les structures argileuses kaoliniques, tout en s'apparentant à celle de la nappe mindélienne, en diffère cependant quelque peu. D'une part, la tranche d'alluvions renfermant une proportion notable de kaolinites est, en règle générale, plus épaisse ( $3 \mathrm{~m}$ environ, contre 1,5 à $2 \mathrm{~m}$ dans le Mindel). D'autre part, ces kaolinites sont souvent un peu plus abondantes, en proportion du stock global de minéraux argileux, dans ces horizons. Enfin, on observe fréquemment une légère amélioration de la cristallinité de ces structures kaoliniques (également proches des métahalloysites), pour des horizons homologues et de profondeur comparable dans le Günz et dans le Mindel. Les diffractogrammes $X$ traduisent assez clairement ce phénomène (figure $14 \mathrm{~d}, \mathrm{e}$ ). On notera enfin que, comme dans le Mindel et le Donau, la goethite (l'hématite, très rarement) se révèle dans la matrice des cailloutis; et, comme dans le Mindel aussi, la gibbsite apparaît au cœur des galets altérés, surtout au sein des horizons profonds, corrélativement à la diminution ou à la disparition des minéraux kaoliniques.

- L'attribution au Günz de la haute terrasse garonnaise ne repose sur aucun argument direct et objecti- vement contrôlable. Mais elle s'inscrit dans la logique du système stratigraphique alpin, entre un Mindel bien typé d'une part, et la nappe culminante que l'on peut rapporter au Donau, d'autre part.

\section{2 - Les formations concrétionnées à grep et les cuiras-} sements ferrugineux

Les formations concrétionnées et indurées par le fer (grep) sont largement répandues dans l'ensemble du piémont nord-pyrénéen, notamment dans le domaine garonnais. Le terme "grep", d'origine vernaculaire (onomatopée évoquant le frottement des instruments aratoires sur le grep), désigne à vrai dire un grand nombre de faciès de concentration et d'individualisation du fer. Ces faciès peuvent être schématiquement regroupés de la façon suivante (J. Hubschman 1975a).

a) Des accumulations lâches sous forme de concrétions et enduits ferromanganiques noirs non jointifs qui, lorsque le sol est sec, confèrent à ces niveaux un surcroît de cohésion d'autant plus sensible que les graviers et gravillons sont abondants. 
b) Des greps tendres, plus ou moins épais, où concrétions, enduits et épigénisations des éléments structuraux et des graviers tendent à devenir jointifs. A l'état $\sec$, ces horizons sont déjà durs et représentent un obstacle mécanique non négligeable pour le travail du sol, l'enracinement et le dynamique hydrique.

c) Des greps durs, épais de 10 à $25 \mathrm{~cm}$ en moyenne, toujours chargés de gravillons, graviers ou galets et qui constituent une aggravation du faciès précédent. A l'état humide, ils se laissent disloquer à la main ou au marteau. Ils présentent généralement une structure pisolithique et, en lame mince, une allure de poudingue de type "nougat" (photo 18).

d) Des noyaux cuirassés, ëpais de 30 à $50 \mathrm{~cm}$ en moyenne, extrêmement indurés, englobant d'abondants éléments grossiers. La structure est plutôt scoriacée que pisolithique, la coupe offrant également l'aspect d'un poudingue (photo 18).

L'arrêt $\mathrm{n}^{\circ} 14$ permet précisément d'étudier un faciès de noyau cuirassé en place. Epaisse d'une cinquantaine de $\mathrm{cm}$, la formation est développée dans le Günz, mais on aurait pu tout aussi bien l'observer localement dans le Mindel. Le ciment qui soude ici graviers et galets dose en moyenne de 15 à $25 \%$ de fer total (exprimé en Fe-métal) et de 1 à quelques $\%$ de manganèse. En profondeur, la cuirasse passe à une formation encore riche en fer mais plus argileuse et meuble à l'état humide, dont la cohésion et la teneur en fer décroissent rapidement vers le bas.

La cuirasse ainsi formée ne couvre d'un seul tenant que des plages peu étendues, discontinues, de l'ordre de la dizaine ou de la centaine de $\mathrm{m}^{2}$ (d'où l'appellation de "noyaux"). Latéralement, elle passe en effet à des greps durs ou tendres, ou même à de simples accumulations lâches.

C'est dire que la genèse de ce type de formations résulte d'une combinaison de facteurs où le pédoclimat et le régime hydrique, commandés par la position dans les toposéquences, tiennent le premier rôle. La quasitotalité des greps et noyaux cuirassés se développe ainsi au sommet de l'alluvion caillouteuse et, plus précisément, au contact des limons et de la grave. C'est là en effet que se manifestent avec le maximum d'intensité et de fréquence les battements de nappes d'eau perchées au sommet des cailloutis. Ces dernières redistribuent et abandonnent éventuellement le fer qu'elles transportent, en priorité dans la zone de battement de nappe. Car cette zone, par sa charge grossière de graviers et galets, par la discontinuité hydrodynamique liée au contact limons/graves, représente un piège géochimique privilégié pour le fer remobilisé. Ceci explique que greps et noyaux cuirassés ne se développent vraiment que lorsque la couverture limoneuse est peu épaisse (moins de $50 \mathrm{~cm}$ ): l'intensité et la fréquence $\mathrm{du}$ battement phréatique se trouvent ainsi favorisées par la proximité de la surface, donc par une plus grande sensibilité aux variations bioclimatiques.

Greps et noyaux cuirassés peuvent donc être assimilés à des sortes de cuirasses de nappe, dont la genèse est étroitement tributaire des conditions stationnelles qui règlent l'économie de l'eau. Il n'en demeure pas moins que, selon l'âge des alluvions, les faciès de concentration du fer ne sont pas développés de façon identique :

. les faciès $a$ et $b$ se retrouvent pratiquement partout là où les situations locales l'autorisent, du Würm (plus rare pour le faciès b) au Donau ;

. le faciès $c$ s'observe fréquemment dans les terrasses antéwürmiennes, exceptionnellement dans le Würm. Un exemple en est donné à l'arrêt $n^{\circ} 21$ (troisième journée, p. 197);

. le faciès $d$ (noyaux cuirassés) enfin, quoique peu répandu, caractérise surtout le Günz et le Mindel mais infiniment moins le Donau, beaucoup mieux drainé en raison de sa dissection avancée et ancienne.

Là encore, il apparaît que le pédoclimat des cailloutis explique fondamentalement la distribution de ces faciès d'un étage à l'autre. Selon que les alluvions caillouteuses sont argilisées et colmatées ou non, en effet, la dynamique des nappes phréatiques perchées au sommet des cailloutis n'est pas la même. Dans le Würm, la perméabilité de la grave très peu argilisée interdit la formation de véritables nappes d'eau perchées: seule s'observe une nappe phréatique libre au toit de la molasse. Déjà, en revanche, la légère argilisation du Riss et l'épaisseur non négligeable des horizons IIB $_{t}$ autorisent l'établissement d'une nappe d'eau diffuse, perchée au sommet du cailloutis et dans les horizons profonds du limon superficiel. D'où la présence, qui n'est pas rare, de greps déjà fortement indurés. Quant aux alluvions antérissiennes, il est clair que leur fort degré d'argilisation et de colmatage induit la formation de nappes d'eau perchées (presque permanentes dans certaines situations), dont les battements fréquents et contrastés constituent le moteur du cuirassement localisé. A cela s'ajoute que, dans ces terrasses, la quantité de fer libéré des minéraux primaires et remobilisé dans les nappes perchées, est, comparée au Riss, très sensiblement supérieure, en rapport avec le degré d'altération poussée du matériel.

\section{III. - LE DONAU DE LA NAPPE CULMINANTE GARONNAISE}

\section{1 - L'évolution pédo-géochimique des alluvions cail- louteuses}

Très dégradée, la nappe culminante garonnaise, on l'a vu, ne forme plus aujourd'hui qu'un ensemble de 
terrains très discontinus : un dense réseau de vallées et vallons l'a réduite en un complexe compartimentage de petits plateaux et d'étroites lanières inclinées vers le fleuve.

L'absence de bonnes coupes compatibles avec le trajet de l'excursion (à l'ouest puis à l'amont de Toulouse) a imposé un bref détour vers le nord, destiné à l'observation d'une carrière ouverte dans la nappe culminante tarnaise (arrêt $\mathrm{n}^{\circ}, 9$ ).

Bien que pratiquement inévitable, le choix de cette coupe n'était pas des plus favorables. D'une part en effet, la stratigraphie des terrasses alluviales tarnaises n'est pas tout à fait identique à celle des remblaiements garonnais. On n'y retrouve pas clairement, en particulier, les deux nappes antémindéliennes garonnaises, si bien que, du point de vue géomorphologique, il n'est qu'une seule génération de terrasse antérieure au Mindel tarnais. Pour A. Tavoso (1975 et communication orale), il s'agirait de l'équivalent du Günz. On pourrait toutefois interpréter cette formation culminante tarnaise, très dégradée et démantelée, comme une nappe polygénique dont les secteurs les plus élevés seraient localement à rapporter au Donau et les zones plus basses au Günz. Ce type de formation complexe et polygénique s'observe en effet assez fréquemment dans la vallée de la Garonne, entre la Save et la confluence Tarn-Garonne.

D'autre part, le bassin d'alimentation tarnais a fourni des matériaux alluviaux sensiblement différents de ceux qui composent les terrasses garonnaises : moins d'éléments gneisso-granitiques et plus de cailloutis quartzeux, en particulier. D'où une certaine difficulté à comparer des profils d'altération développés dans l'un ou l'autre des deux bassins.

Il reste que, par nombre de paramètres fondamentaux, la carrière présentée à l'arrêt $\mathrm{n}^{\circ} 9$ demeure très proche des profils différenciés dans le Donau garonnais. Néanmoins, les problèmes soulevés étant les mêmes, on a jugé préférable de décrire et de commenter ici une coupe de la nappe culminante garonnaise. Elle est située entre Save et Gimone, à proximité d'Escazeaux (c'est-à-dire à une latitude voisine de celle de l'arrêt $n^{\circ} 9$ ) et en bordure d'une lanière de plateau, vers $250 \mathrm{~m}$ d'altitude.

\section{Description sommaire du profil d'Escazeaux}

Sol lessivé hydromorphe, profil d'altération fersiallitique.

- 0-8 cm : Ap, structure massive, sous-structure granulaire au sommet, racines, pores, galeries, graviers et gravillons TRA 2 à $5 \%$, limite inférieure graduelle, ondulée ;
- 8-25 cm: $A_{2}, 10$ YR 6/2 à 10 YR 7/3, structure massive légèrement feuilletée, pores, canaux, racines, gravillons et graviers TRA $5 \%$, limite inférieure assez franche, ondulée ;

- $2540 \mathrm{~cm}: B_{1 \mathrm{tg}}, 10$ YR $6 / 3$ à 7,5 YR 6/4, structure finement polyédrique, graviers, gravillons et petits galets TRA 20 à $30 \%$, revêtements argileux, concrétions noires TRA 5 à $10 \%$ limite inférieure variable : graduelle ou franche, ondulée ;

$-40-70 \mathrm{~cm}:$ IIB $_{2 \mathrm{tg}}$, nappe de galets, graviers et gravillons TRA 30 à $50 \%$, quartz dominants, matrice fond 10 YR $7 / 3$ à larges plages 7,5 YR 6/6 à 10 YR $6 / 4$, revêtements argileux et enduits et concrétions noirs, limite inférieure graduelle, ondulée, transition rapide ;

- 70-110 cm: IIB $_{3 \text { tg }}$, idem mais matrice plus marmorisée fond 5 YR 5/6 TRA $70 \%$ et plages claires 7,5 YR 5/0 à 10 YR 6/2, organisation subhorizontale des zones de couleur, nombreux gravillons de quartz non roulés, nombreux éléments quartzitiques silicifiés à cortex, limite inférieure graduelle ;

$-110-200 \mathrm{~cm}: \operatorname{IIB}_{4 \mathrm{tg}}$ (à $\mathrm{IIBC}_{1 \mathrm{~g}}$ ), idem mais très nette organisation horizontale des zones de couleur, succession de bandes à fond 2,5 YR 3/6 à 5 YR 5/6 TRA 30 à $50 \%$ et de bandes à fond 7,5 YR $8 / 0$ à 7,5 YR 7/0 TRA 50 à $70 \%$, reliées par des bretelles obliques, panachage généralisé des galets et graviers, enduits noirs locaux, limite inférieure graduelle, irrégulière ;

$-200-270 \mathrm{~cm}:$ IIB $_{5 t g}\left(\right.$ à $\left.\mathrm{IIBC}_{2 \mathrm{~g}}\right)$, idem mais organisation horizontale moins apparente, matrice à fond vif 2,5 YR $4 / 6$ à 5 YR $5 / 4$ dominante (TRA $80 \%$ ), limite inférieure graduelle;

$-270-500 \mathrm{~cm}: \mathrm{IIBC}_{3 \mathrm{~g}}$, nettement moins marmorisé, matrice fond $2,5 \mathrm{YR} 5 / 6$ à 5 YR $5 / 6$ devenant 7,5 YR 6/8 à 7,5 YR 5/6 en profondeur, rares plages grises, quelques amas poudreux blanchâtres à débris quartzeux en profondeur, limite inférieure très graduelle ;

- 500-700 cm: IIBC $_{4 \mathrm{~g}}$, idem mais matrice plus grossière et plus terne fond $5 \mathrm{YR} 5 / 4$, amas poudreux blanchâtres, fantômes exceptionnels de cristallins, limite inférieure très graduelle ;

$-700-850 \mathrm{~cm}:$ IIBC $_{5 \mathrm{~g}}$, idem mais amas poudreux plus fréquents, rares fantômes de cristallins et par place très rares galets de granite totalement pulvérisés mais identifiables, limite inférieure très graduelle ;

- 850-1 $000 \mathrm{~cm}: \mathrm{IIBC}_{6 \mathrm{~g}}$, le plancher de la carrière est toujours dans l'alluvion, idem ci-dessus. 


\begin{tabular}{|c|c|c|c|c|c|c|}
\hline \multirow{3}{*}{$\begin{array}{l}\text { Prof. } \\
\mathrm{cm}\end{array}$} & \multicolumn{6}{|c|}{ Granulométrie \% } \\
\hline & \multirow[b]{2}{*}{ EG } & \multicolumn{5}{|c|}{$\%$ de TF } \\
\hline & & SG & $\mathrm{SF}$ & STF & L & A \\
\hline $10-20$ & 23,7 & 7,7 & 9,6 & 35,4 & 23,1 & 21,4 \\
\hline $30-40$ & 68,8 & 11,0 & 8,9 & 21,5 & 23,3 & 33,9 \\
\hline $55-65$ & 73,9 & 18,2 & 7,7 & 18,9 & 18,6 & 35,3 \\
\hline $90-100$ & 77,2 & 18,9 & 6,0 & 5,1 & 8,4 & 60,9 \\
\hline $130-140$ & 75,0 & 41,6 & 8,5 & 3,4 & 8,6 & 37,2 \\
\hline $150-160$ & 68,1 & 17,6 & 6,3 & 3,3 & 7,2 & 64,7 \\
\hline $230-240$ & 66,1 & 45,9 & 8,9 & 3,9 & 11,3 & 29,2 \\
\hline $280-300$ & 76,5 & 51,3 & 9,9 & 3,7 & 10,4 & 24,3 \\
\hline $400-420$ & 67,3 & 47,3 & 7,5 & 3,6 & 7,8 & 32,8 \\
\hline $620-640$ & 71,4 & 49,8 & 10,4 & 4,0 & 9,7 & 23,5 \\
\hline $780-800$ & $69 ; 7$ & 52,4 & 7,3 & 2,3 & 6,8 & 28,1 \\
\hline
\end{tabular}

\begin{tabular}{|c|c|c|c|c|c|c|}
\hline \multirow{2}{*}{$\begin{array}{c}\text { Prof. } \\
\mathrm{cm}\end{array}$} & \multicolumn{2}{|c|}{$\mathrm{pH}$} & \multicolumn{4}{|c|}{ Matière organique \% } \\
\cline { 2 - 7 } & eau & $\mathrm{KCl}$ & $\mathrm{MO}$ & $\mathrm{C}$ & $\mathrm{N}$ & $\mathrm{C} / \mathrm{N}$ \\
\hline $10-20$ & 6,9 & 5,7 & 1,6 & 0,98 & 0,11 & 8,8 \\
$30-40$ & 6,6 & 5,4 & 0,9 & 0,53 & 0,08 & 6,08 \\
$55-65$ & 6,1 & 4,9 & 0,4 & 0,25 & 0,05 & 4,3 \\
$90-100$ & 5,2 & 4,0 & 0,3 & 0,23 & 0,05 & 4,4 \\
$130-140$ & 4,8 & 3,9 & 0,1 & 0,08 & 0,03 & 2,4 \\
$150-160$ & 4,8 & 3,7 & 0,2 & 0,12 & 0,04 & 2,8 \\
$230-240$ & 4,8 & 3,9 & 0,06 & 0,04 & 0,02 & 1,4 \\
$280-300$ & 5,0 & 4,3 & 0,06 & 0,04 & 0,03 & 1,3 \\
$400-420$ & 4,6 & 4,6 & 0,06 & 0,04 & 0,02 & 1,4 \\
$620-640$ & 4,8 & 3,8 & & & & \\
$780-800$ & 4,9 & 3,8 & & & & \\
\hline
\end{tabular}

\begin{tabular}{|c|c|c|c|c|c|c|c|c|c|c|}
\hline \multirow{2}{*}{$\begin{array}{c}\text { Prof. } \\
\mathrm{cm}\end{array}$} & \multicolumn{9}{|c|}{ Complexe absorbant meq \% } & \multicolumn{4}{c|}{ Fe \% } \\
\cline { 2 - 10 } & $\mathrm{Ca}$ & $\mathrm{K}$ & $\mathrm{Mg}$ & $\mathrm{Na}$ & $\mathrm{S}$ & $\mathrm{T}$ & $\mathrm{S} / \mathrm{T} \%$ & $\mathrm{FT}$ & $\mathrm{FL}$ & FL/FT \% \\
\hline \multirow{2}{*}{20} & 7,7 & 0,23 & 0,65 & 0,12 & 8,7 & 10,9 & 79 & 1,34 & 0,67 & 50 \\
$30-40$ & 7,7 & 0,21 & 0,60 & 0,16 & 8,8 & 12,0 & 73 & 1,95 & 1,06 & 54 \\
$55-65$ & 7,1 & 0,14 & 1,1 & 0,12 & 8,6 & 13,0 & 66 & 2,63 & 1,39 & 53 \\
$90-100$ & 9,5 & 0,12 & 1,8 & 0,16 & 11,7 & 20,4 & 57 & 7,20 & 4,18 & 58 \\
$130-140$ (a) & 4,2 & 0,04 & 1,6 & 0,12 & 6,0 & 14,40 & 42 & 3,01 & 2,00 & 65 \\
$130-140$ (b) & & 0,08 & 1,7 & 0,19 & 9,8 & 21,1 & 46 & 10,15 & 6,08 & 60 \\
$150-160$ (c) & 7,8 & 0,08 & 0,72 & 36 \\
$230-240$ (d) & 3,0 & 0,04 & 1,1 & 0,12 & 4,3 & 9,9 & 43 & 3,62 & 2,90 & 80 \\
$280-300$ (e) & 3,1 & 0,04 & 1,1 & 0,09 & 4,4 & 8,7 & 50 & 3,29 & 2,51 & 76 \\
$400-420$ (f) & 4,1 & 0,17 & 1,4 & 0,12 & 5,9 & 9,2 & 64 & 3,40 & 2,73 & 80 \\
\hline
\end{tabular}
(a) : Dominante grise
(b) : Dominante rouge pure
(c) : Dominante grise pure
(d) (e) (f) : Dominante rouille ou ocre
(g) : 3 attaques

\begin{tabular}{|c|c|c|c|c|}
\hline $\begin{array}{l}\text { Prof. } \\
\mathrm{cm}\end{array}$ & Illites & $\mathrm{C}-\mathrm{V}-\mathrm{Al}$ & Kaolinites & $\begin{array}{c}\text { Interstratifiés } \\
\text { gonflants }\end{array}$ \\
\hline $\begin{array}{r}10-20 \\
30-40 \\
90-100 \\
150-160 \\
230-240 \\
280-300 \\
400-420 \\
620-640 \\
780-800\end{array}$ & $\begin{array}{c}\mathrm{M} \\
\mathrm{F} \\
\mathrm{M} \\
\mathrm{f} \\
\mathrm{m} \\
\mathrm{M} \\
\mathrm{M} \\
\mathrm{M} \\
\mathrm{m}\end{array}$ & $\begin{array}{l}\mathrm{F} \\
\mathrm{M} \\
\mathrm{m} \\
\mathrm{M} \\
\mathrm{F} \\
\mathrm{m} \\
\mathrm{f} \\
\mathrm{m} \\
\mathrm{M}\end{array}$ & $\begin{array}{c}\mathrm{f} \\
\mathrm{m} \\
\mathrm{M} \\
\mathrm{F} \\
\mathrm{M} \\
\mathrm{F} \\
\mathrm{F} \\
\mathrm{M} \\
\mathrm{F}\end{array}$ & $\begin{array}{l}\text { f } \\
\text { f } \\
\text { tr }\end{array}$ \\
\hline
\end{tabular}


- L'organisation générale du profil, dans la nappe culminante, diffère très sensiblement de celle qui marque les remblaiements postérieurs, günziens et mindéliens. Hormis la différenciation de la couverture limoneuse, ici vigoureusement tronquée et plus ou moins mélée à la grave (situation en bordure de lanière de plateau), il est clair que les différentes séquences progressives sont considérablement transformées, en raison même de la puissance de l'altération. Celle-ci affecte toute la tranche visible de la coupe, l'épaisseur du remblaiement alluyial avoisinant ici 12 à $15 \mathrm{~m}$ au-dessus de la molasse.

- La séquence progressive d'appauvrissement de la matrice en particules argileuses se déploie sur toute l'épaisseur du profil, les taux d'argile atteignant encore couramment 25 à $35 \%$ entre 8 et $10 \mathrm{~m}$ de profondeur. On note en même temps une certaine irrégularité de cette séquence, surtout vers le sommet de l'alluvion caillouteuse : cette irrégularité est sans doute liée en partie aux migrations obliques de particules ou de solutions ioniques.

La séquence progressive d'enrichissement en galets cristallins gneisso-granitiques, comparée à celles du Günz et du Mindel, offre un faciès tout à fait spécifique. Ici, en effet, ces éléments ne sont pratiquement plus reconnaissables et n'apparaissent qu'exceptionnellement dans les horizons les plus profonds du profil. La séquence existe cependant, révélée par l'abondance, croissante avec la profondeur, d'amas poudreux blanchâtres intégrés à la matrice (mélange d'argiles, de quartz et de feldspaths potassiques) ou, dans le meilleur des cas, de fantômes de galets cristallins pulvérisés et englobés dans la matrice (figure 15). On notera toutefois que dans ce profil, situé dans un secteur déjà éloigné des Pyrénées, la taille des éléments caillouteux reste modeste, n'excédant guère 6 à $8 \mathrm{~cm}$, comme d'ailleurs dans la carrière de l'arrêt $n^{\circ} 9$. Ce caractère a dû faciliter une "digestion" plus complète des galets cristallins : à l'amont de Toulouse, en effet, la proportion de fantômes gneisso-granitiques identifiables est un peu plus élevée dans les horizons profonds, en rapport avec la dimension plus importante des galets. Concernant la coupe de l'arrêt $n^{\circ} 9$, il faut également rappeler que les alluvions tarnaises sont initialement moins riches en éléments gneisso-granitiques que leurs homologues garonnaises, ce qui renforce d'autant ce phénomène.

- La dynamique du fer, dans les remblaiements danubiens, se marque surtout par l'organisation très apparente, parfois même caricaturale, des zones de redistribution du fer. Le sommet de la nappe caillouteuse fixe tout particulièrement l'attention, par l'alternance de bandes subhorizontales très pâles et très vivement colorées qu'il présente et par l'éclatant contraste entre ces colorations. Le phénomène est iden- tique à celui du Mindel ou du Günz, mais en plus poussé (à conditions égales de situation topographique, de drainage et de position dans les toposéquences).

Une fois encore, les faciès de rubéfaction se trouvent brouillés par cette intense redistribution du fer, lequel colore localement en rouge vif certaines bandes de dégradation. Toutefois, cette coloration est souvent plus vive que dans les profils du Günz ou du Mindel. D'autre part, les alluvions sous-jacentes au sommet du cailloutis, moins affectées par la dégradation en bandes, montrent une matrice de teinte touille ou rougeâtre plus soutenue que dans le Günz ou le Mindel, avec des taux de libération du fer habituellement un peu plus élevés.

Enfin, on note l'abondance de cortex blancs ou rouille des quartzites, souvent à faciès de silex, parfois presque totalement blanchis ou rubéfiés selon leur position dans la matrice claire ou colorée. Plus à l'amont, la dimension des quartzites est sensiblement supérieure et les cortex moins fréquents et surtout moins épais relativement à la taille des galets.

- L'ambiance ionique des cailloutis danubiens diffère également de celle qui baigne les remblaiements mindéliens et günziens. Ici, sous le profil superficiel et le sommet de la grave qui le prolonge, la séquence des pH est plus régulière que dans le Günz et le Mindel. En outre et surtout, ces $\mathrm{pH}$ fluctuent très généralement entre 4,5 et 5: la mosaique de $\mathrm{pH}$ est ici singulièrement moins mouvante et plus acide que dans les remblaiements postérieurs, ce dont l'interprétation des néoformations argileuses devra tenir compte (cf. p. 212).

- La séquence minéralogique argileuse de la matrice, contrairement à celles du Günz et du Mindel, ne traduit pas de gradient lié à la profondeur. D'une part, les minéraux de la famille des kaolinites sont plus abondants à tous les niveaux (de $1 / 3$ à $2 / 3$ environ du stock global d'argiles), sans qu'on puisse observer une quelconque loi de distribution verticale. D'autre part, ces minéraux kaoliniques sont beaucoup mieux organisés, du point de vue de la cristallinité du réseau, que dans le Günz et le Mindel, comme le montre leur définition diffractométrique (figure $14 \mathrm{f}, \mathrm{g}, \mathrm{h}$ ) ; ce caractère de cristallinité n'évolue pas sensiblement avec la profondeur. Enfin, si la goethite apparaît fréquemment mêlée aux argiles, aucune trace de gibbsite n'est décelable ni dans la matrice, ni dans les résidus de galets gneisso-granitiques pulvérisés.

- L'attribution au Donau de la nappe culminante garonnaise repose sur l'identité de faciès entre cette demière et les alluvions rapportées au Donau par H. Alimen (1964) dans les Pyrénées occidentales, alluvions dont les caractères ont été précisées par la suite par M. Icole (1969a, 1973) (cf. p. 207). 


\section{2 -Origine et différenciation des couvertures limo- neuses}

La nappe culminante garonnaise, on l'a vu, domine l'ensemble du paysage alluvial et molassique. De fait, lorsque l'on quitte la Forêt de Bouconne (arrêts 12, 13, 14) par Pujaudran, la route D 58 suit le sommet du balcon que forme le Donau au-dessus de la Gascogne gersoise, dont les sommets s'inscrivent 30 à $40 \mathrm{~m}$ plus bas vers l'ouest. L'inversion de relief, déjà évoquée dans les pages précédentes (cf. p. 130) et qui s'est traduite par un vigoureux rajeunissement du modelé molassique, apparaît ici dans toute son ampleur.

Entre Bouconne et Petites Pyrénées, la nappe culminante offre toujours son classique aspect discontinu : étroites lanières de plateau séparées de profonds vallons et généralement drapées d'un manteau limoneux superficiel plus ou moins épais.

L'évolution de ces limons, comme il a déjà été dit, ne diffère pas sensiblement de celle des couvertures du Günz et du Mindel. Toutefois, les limons du Donau garonnais montrent des faciès glossiques un peu plus fréquents et plus nettement exprimés que dans les manteaux superficiels des terrasses postérieures. L'arrêt $\mathrm{n}^{\circ} 15$, à proximité d'Empeaux et Saint-Thomas, en est une illustration.

\section{Description sommaire du profil proche de l'arrêt $n^{\circ} 15$}

Sol lessivé (hydromorphe) glossique

- 0-15 cm : Ap, brun clair, quelques taches rouille, structure polyédrique légèrement litée, racines, limite inférieure graduelle mais transition rapide, ondulée ;

$-15.40 \mathrm{~cm}: A_{2 g}$ et $B_{1 \mathrm{tg}}$, fond 10 YR $7 / 3$ avec plages ou points 10 YR $8 / 2$ et 10 YR $6 / 8$ plus nets en profondeur, structure massive, sous-structure légèrement feuilletée puis finement polyédrique, limite inférieure graduelle. irrégulière ;

- 40-60 cm: $B_{2 \operatorname{tg}}$, fond 10 YR $6 / 8$ TRA $60 \%$ et plages 10 YR $8 / 2$, quelques concrétions et enduits noirs, structure polyédrique, revêtements argileux abondants, limite inférieure graduelle, ondulée ;

- 60-90 cm: $\mathrm{B}_{3 \mathrm{tg}}$, fond 10 YR $7 / 6$ TRA $50 \%$ et zones 10 YR $8 / 2$ tendant à s'organiser peu à peu en langues subverticales, revêtements argileux, concrétions et enduits noirs, limite inférieure graduelle ;

- 90-130 cm: $B_{4 t g}$, idem mais disposition glossique mieux exprimée par langues grises 10 YR $8 / 2$, larges et anastomosées, bordées de liserés rouille vif et se prolongeant en profondeur.

\begin{tabular}{|c|c|c|c|c|c|c|}
\hline \multirow{2}{*}{$\begin{array}{c}\text { Prof. } \\
\text { cm }\end{array}$} & \multicolumn{5}{|c|}{ Granulométrie \% } \\
\cline { 2 - 7 } & EG & SG & SF & STF & L & A \\
\cline { 2 - 7 } & 2,4 & 9,1 & 14,9 & 27,3 & 24,2 & 21,0 \\
$15-30$ & 0 & 6,2 & 12,6 & 25,8 & 23,7 & 28,6 \\
$40-60$ & 0 & 4,4 & 9,0 & 26,3 & 23,9 & 35,4 \\
$60-80$ & 0 & 4,8 & 10,5 & 25,4 & 23,4 & 34,7 \\
$110-120$ & 0 & 6,2 & 11,8 & 22,9 & 23,0 & 34,9 \\
\hline
\end{tabular}

\begin{tabular}{|c|c|c|c|c|c|c|c|c|}
\hline \multirow{2}{*}{$\begin{array}{c}\text { Prof. } \\
\mathrm{cm}\end{array}$} & \multicolumn{2}{|c|}{$\mathrm{pH}$} & \multicolumn{2}{c|}{$\mathrm{P}_{2} \mathrm{O}_{\mathbf{5}} \%$} & \multicolumn{4}{c|}{ Matière organique \% } \\
\cline { 2 - 9 } & eau & $\mathrm{KCl}$ & citrique & Joret & $\mathrm{MO}$ & $\mathrm{C}$ & $\mathrm{N}$ & $\mathrm{C} / \mathrm{N}$ \\
\hline $15-30$ & 6,5 & 5,2 & 0,02 & 0,02 & 2,0 & 1,16 & 0,13 & 8,7 \\
$40-60$ & 6,7 & 5,3 & 0,00 & 0,00 & 0,3 & 0,22 & 0,04 & 4,7 \\
$60-80$ & 6,6 & 5,1 & 0,00 & 0,00 & 0,3 & 0,17 & 0,05 & 3,3 \\
$110-120$ & 5,5 & 3,7 & 0,00 & 0,00 & 0,2 & 0,12 & 0,04 & 2,7 \\
\hline
\end{tabular}

\begin{tabular}{|c|c|c|c|c|c|c|c|c|c|c|}
\hline & \multicolumn{9}{|c|}{ Complexe absorbant meq \% } & \multicolumn{4}{c|}{ Fe \% } \\
\cline { 2 - 11 } $\begin{array}{c}\text { Prof. } \\
\mathrm{cm}\end{array}$ & $\mathrm{Ca}$ & $\mathrm{K}$ & $\mathrm{Mg}$ & $\mathrm{Na}$ & $\mathrm{S}$ & $\mathrm{T}$ & $\mathrm{S} / \mathrm{T} \%$ & $\mathrm{FT}$ & $\mathrm{FL}$ & $\mathrm{FL} / \mathrm{FT} \%$ \\
\hline $15-30$ & 10,7 & 0,23 & 1,6 & 0,09 & 12,7 & 14,5 & 87 & 2,23 & 1,10 & 50 \\
$40-60$ & 10,2 & 0,23 & 2,2 & 0,12 & 12,8 & 16,1 & 79 & 2,95 & 1,22 & 41 \\
$60-80$ & 8,7 & 0,17 & 3,9 & 0,16 & 13,0 & 15,2 & 85 & 2,90 & 1,28 & 44 \\
$110-120$ & 4,9 & 0,12 & 4,5 & 0,35 & 9,9 & 14,4 & 68 & 2,95 & 1,39 & 47 \\
\hline
\end{tabular}




\begin{tabular}{|c|c|c|c|c|}
\hline $\begin{array}{l}\text { Prof. } \\
\mathrm{cm}\end{array}$ & Illites & $\mathrm{C}-\mathrm{V}-\mathrm{Al}$ & Kaolinites & $\begin{array}{c}\text { Interstratifiés } \\
\text { gonflants }\end{array}$ \\
\hline $\begin{array}{r}5-10 \\
15-30 \\
40-60 \\
60-80 \\
110-120\end{array}$ & $\begin{array}{l}\mathrm{F} \\
\mathrm{F} \\
\mathrm{M} \\
\mathrm{M} \\
\mathrm{M}\end{array}$ & $\begin{array}{l}\mathrm{M} \\
\mathrm{M} \\
\mathrm{F} \\
\mathrm{F} \\
\mathrm{F}\end{array}$ & $\begin{array}{l}\mathrm{f} \\
\mathrm{tr} \\
\mathrm{f}\end{array}$ & $\begin{array}{c}\mathrm{f} \\
\mathrm{tr} \\
\mathrm{f}\end{array}$ \\
\hline
\end{tabular}

A) Evolution pédologique des limons.

Le faciès glossique, particulièrement bien exprimé ici (photo 19), constitue la note dominante de ce type de profil. On y observe la "dégradation" déjà sensible au sommet du profil ("descente" du $\mathrm{A}_{2 \mathrm{~g}}$ dans le $\mathrm{B}_{\mathrm{tg}}$ ) et l'intense redistribution du fer dès la surface.

La différenciation texturale n'est pas très poussée, caractère assez général dans les sols glossiques. On remarquera toutefois l'absence de "ventre" dans la distribution verticale de l'argile : la totalité du matériau limoneux est transformée en $B_{t g}$, parfois sur $2 \mathrm{~m}$ et plus, lorsque le limon offre une puissance de cet ordre.

L'organisation en glosses reflète essentiellement la redistribution $\mathrm{du}$ fer : très faibles teneurs dans les langues grises, fortes teneurs dans les gaines rouille vif, teneurs moyennes dans le fond général brun-jaune ocre. L'observation micromorphologique ne permet pas de discriminer clairement ces différentes zones par des caractères autres que ceux liés à la redistribution du fer.

L'évolution des minéraux argileux s'apparente étroitement à celle qui marque toutes les couvertures limoneuses lessivées hydromorphes antéwürmiennes ou antérissiennes.

Notons, en dernier lieu, que les limons glossiques, bien qu'un peu plus fréquents au sommet du Donau, sont loin d'être rares sur les terrasses postérieures. Encore peu répandus et localisés sur le niveau rissien,

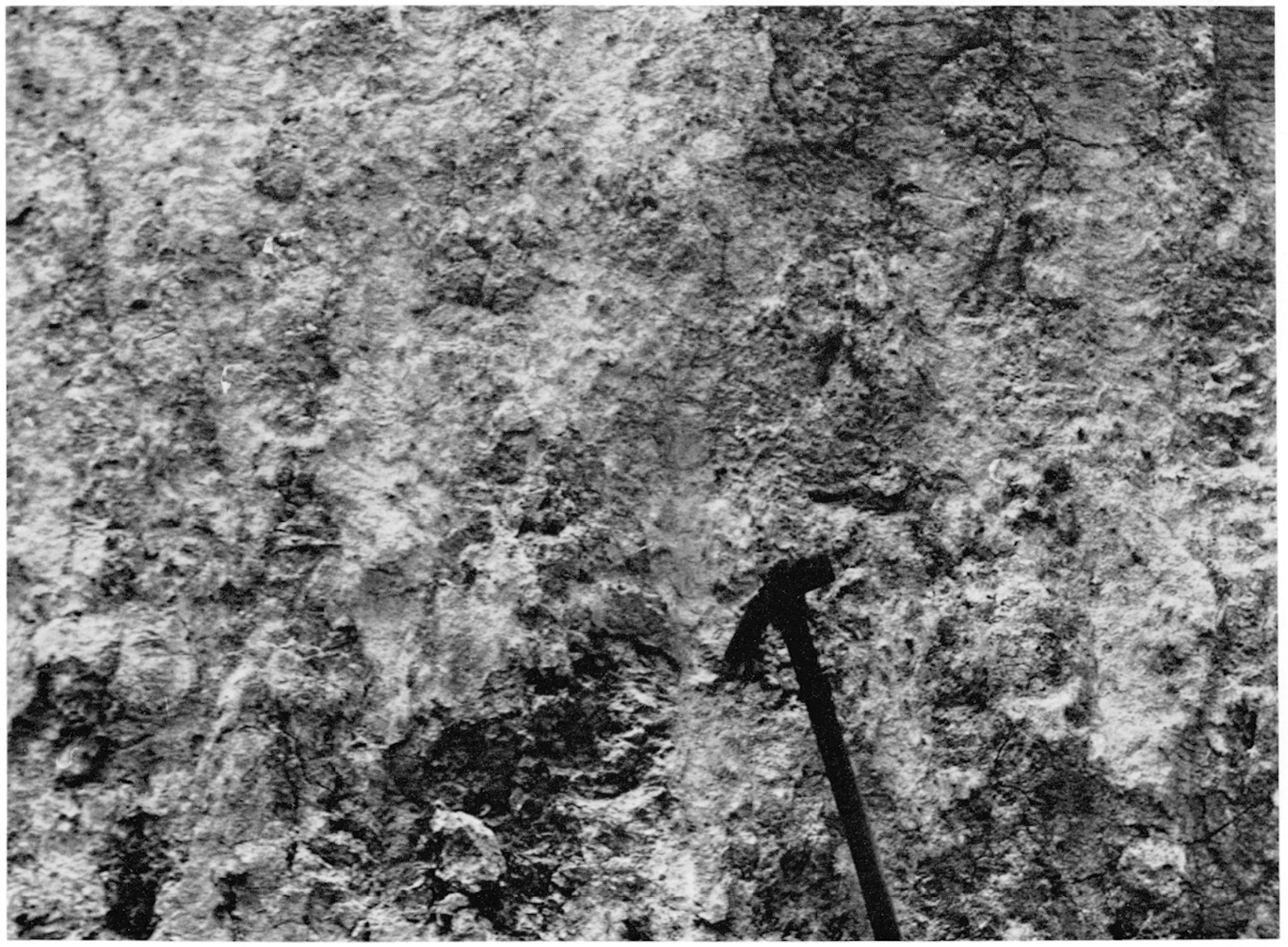

Photo 19 - Faciès glossique des sols lessivés hydromorphes développés dans les couvertures limoneuses du Donau (vers $1 \mathrm{~m}$ de profondeur). 
ils abondent en revanche sur le Mindel et le Günz. Dans tous les cas, ces limons glossiques se développent d'autant mieux que les conditions stationnelles le permettent : épaisseur du limon, position dans les toposéquences et drainage interne relativement moins médiocre qui en résulte (J.C. Bégon 1972, J.C. Bégon et M. Jamagne 1972, J. Hubschman 1975a).

B) Age, origine et mode de mise en place des limons.

Il s'agit ici d'un problème-clé. On aborde ainsi l'ensemble de la problématique que soulève l'existence générale de ces manteaux limoneux dans le piémont nord-pyrénéen, et dont les limons du Donau garonnais constituent une illustration exemplaire. L'arrêt $n^{\circ} 15$, à cet égard, a fourni l'occasion d'une discussion au cours de laquelle ce sont deux points de vue, pour l'essentiel, qui ont été confrontés.

- Le premier corps d'explication s'appuie surtout sur le caractère allochtone du matériau limoneux et notamment sur sa mise en place éolienne à une époque relativement récente. Schématiquement, les principaux arguments sont les suivants :

a) La vulnérabilité à l'érosion des limons interdit de penser que, mis en place à une période très reculée, ils aient pu se conserver jusqu'à aujourd'hui. Ces couvertures sont donc très postérieures au niveau ancien qu'elles revêtent.

b) Dès la terrasse rissienne et surtout dès le Mindel, l'évolution pédologique et géochimique des limons reste à peu près identique d'un étage à l'autre, lorsque l'on remonte les temps quaternaires. Ainsi, les limons de l'arrêt $\mathrm{n}^{\circ} 15$ ne peuvent dater du Donau, sinon, ils offriraient une différenciation beaucoup plus poussée : $A_{2}$ épais extrêmement apprauvris, rubéfaction prononcée, minéraux argileux etc.

c) Il en résulte que les manteaux limoneux sont relativement jeunes, sans doute mindéliens au plus tôt et würmiens au plus tard, et qu'ils ne peuvent provenir, pour une large part, que d'apports éoliens. A cet égard, l'origine éolienne n'est pas la seule possibilité qui a pu être invoquée : pour certains auteurs dont $M$. Icole (1973), les limons qui couvrent les terrasses prérissiennes proviendraient surtout d'un vigoureux remaniement périglaciaire de la matrice fine des cailloutis sous-jacents, redistribuée et étalée en surface au cours du Riss et du Würm.

- Le second point de vue, que nous défendons, revient à considérer que, sur chaque niveau de terrasse, les limons représentent la phase terminale de la sédimentation alluviale. Aux remaniements près, ils possèdent donc, grosso modo, le même âge que les remblaiements caillouteux qu'ils recouvrent. L'argumentation s'oppose ainsi, point par point, aux propositions précédentes : a) Il n'existe aucune preuve objective que la totalité des limons anciens (c'est-à-dire déposés par exemple au Donau, au Günz ou au Mindel) ait disparu par érosion. On ne voit pas très clairement pourquoi, en particulier, les niveaux günziens et plus encore mindéliens, auraient été débarrassés aussi facilement de leur manteau limoneux, alors que leurs terrasses offrent aujourd'hui encore de vastes étendues pratiquement planes, indemnes de toute trace d'érosion géomorphologiquement appréciable. Là où, en revanche, les terrasses ont été soumises à des facteurs érosifs de quelque efficacité (bordures de vallons, talus des paliers etc.), les limons originels (et bien souvent aussi la grave sous-jacente) ont été effectivement décapés et éventuellement remplacés par un manteau colluvionné de faciès sensiblement différent des couvertures limoneuses habituelles (forte charge d'éléments issus du cailloutis sousjacent, teneur non négligeable en minéraux kaoliniques provenant du mélange avec la matrice des graves...). C'est justement ce qui s'est produit pour la nappe culminante du Donau : celle-ci ne subsiste plus que sous forme d'échines résiduelles, sortes de buttes - témoins entre lesquelles l'érosion s'est exercée à plein. Ces buttes-témoins ne couvrent plus, aujourd'hui, qu'une étendue dérisoire au regard de la surface initialement occupée par le niveau danubien. On peut donc parfaitement admettre que, au sommet de ces reliques du Donau, les limons aient pu parvenir jusqu'à nous sinon en l'état, du moins sans bouleversement considérable.

b) L'analogie d'évolution pédologique et géochimique présentée par ces limons d'âge différent, depuis le Riss ou le Mindel jusqu'au Donau, peut s'expliquer par la combinaison de plusieurs paramètres (J. Hubschman 1975 a) :

- D'une part, Porigine molassique de la quasitotalité des limons, au nord des Petites Pyrénées, joue un rôle considérable. La molasse, aboutissement de longues évolutions tertiaires sur les versants pyrénéens et dans le Bassin Aquitain, constitue déjà une rochemère géochimiquement mûre. Elle ne correspond plus, en première analyse, qu'à un mélange de quartz, de calcaire et d'argiles, assorti de quelques minéraux primaires altérables (surtout micas muscovites). Il en résulte que, contrairement aux alluvions caillouteuses sous-jacentes, les limons sont dotés d'une forte inertie géochimique. En l'absence d'abondants minéraux primaires altérables, l'évolution ne peut se manifester que par des processus "classiques": décalcarisation, soustraction ou addition de bases, redistribution du fer, dégradation et agradation des minéraux argileux... L'absence de structures argileuses néoformées comme les kaolinites et la transformation relativement ménagée du stock initial de minéraux argileux ne représentent pas nécessairement un critère de jeunesse, dès lors que les bioclimats pédogénétiques régionaux du Quaternaire ancien et moyen ne sont pas systématiquement consi- 
dérés comme ayant été particulièrement agressifs (thèse qui sera reprise plus loin, cf. p. 211). Dans cette perspective, on comprend que, après une série de transformations relativement rapides correspondant aux premières étapes de l'évolution, celle-ci ne peut que rester pratiquement bloquée à un certain stade géochimique, qui va peu ou prou perdurer jusqu'à aujourd'hui. On s'explique ainsi que, dès la terrasse rissienne, le complexe géochimique des couvertures limoneuses antérissiennes ne se modifie plus guère avec le temps.

- D'autre part, c'est la combinaison d'un matériau parental fin avec les conditions de topographie plane et l'hydromorphie qu'elles induisent, qui constitue le paramètre fondamental de la différenciation des limons. L'évolution des profils limoneux préwürmiens est en effet commandée, dès la surface, par le jeu des processus d'hydromorphie. Dans un type de matériel comme les limons, ces processus interdisent évidemment le développement de puissants horizons éluviaux à dominante squelettique, comme il est possible d'en observer ailleurs dans des formations plus grossières et/ou beaucoup mieux drainées (Bas-Languedoc ou domaine rhodanien, par exemple). Par contre, dans toutes les situations locales conduisant à la différenciation de planosols bien exprimés, les profils montrent effectivement un horizon superficiel, épais de 20 à $30 \mathrm{~cm}$, pratiquement réduit à un squelette quartzeux, totalement blanchi, à points rouille et noirs, et débarrassé de presque tout son fer et ses particules argileuses (J.C. Bégon 1972, J.C. Bégon et M. Jamagne 1972) (1). De la même façon que pour l'évolution géochimique, l'évolution proprement pédologique de ces matériaux limoneux reste bloquée au stade des sols lessivés hydromorphes, glossiques ou planosoliques selon leur position dans les toposéquences. On s'explique ainsi le fait que, à partir du niveau rissien ou mindélien, la différenciation pédologique des couvertures limoneuses antérieures ne montre guère de caractères fondamentalement nouveaux. Le tableau ci-dessous (partie droite du tableau) représente très schématiquement ce phénomène.

(1) Les contraintes matérielles (horaire, itinéraire et coupes) n'ont malheureuscment pas permis l'observation directe de ces planosols au cours de l'excursion AFEQ 1975.
- Enfin, le remaniement plus ou moins important qui a très probablement affecté à plusieurs reprises les couvertures meubles limoneuses au cours de leur histoire (mobilisation par le ruissellement superficiel, mouvements liés au dynamisme interne et à l'activité biologique etc. sans compter les actions anthropiques historiques), ce remaniement a sans doute joué un certain rôle dans le développement des profils. Quelles qu'aient été les causes de ces redistributions de matière (périglaciaires ou non, par exemple), il est clair qu'elles ont contribué à perturber, donc à "rajeunir" et en quelque sorte à homogénéiser l'ensemble des profils différenciés (1).

c) L'origine éolienne de ces limons ne peut être prouvée par des arguments directs et contrôlables. Tout au contraire, l'étude sédimentologique (distribution fréquentielle des sables et exoscopie des grains de quartz) infirme plutôt ce point de vue et plaide en faveur d'une mise en place fluviatile ou par ruissellement (figure 17 et photos 20 et 21 . Concernant l'exoscopie et l'absence de traces de chocs éoliens visibles sur les grains de quartz, on a objecté, avec raison semble-t-il, que la faible dimension des poussières apportées par le vent interdisait la formation de cicatrices de chocs. L'hypothèse est plausible mais on doit alors se demander pourquoi, dans les secteurs de la basse plaine würmienne qui ont été manifestement éolisés (cf. figure 3), les nombreuses traces de chocs apparaissent de façon parfaitement claire à la surface des sables quartzeux de taille comprise entre 0,200 et $2 \mathrm{~mm}$ (J. Hubschman et L. Le Ribault 1972). Or, cette fraction sableuse grossière existe aussi bien dans tous les manteaux limoneux des terrasses antéwürmiennes.

Enfin, la thèse selon laquelle l'essentiel des couvertures limoneuses antérissiennes proviendrait d'un remaniement et d'une redistribution de la matrice fine des cailloutis altérés (M. Icole 1973), cette thèse ne semble

(1) De ces probables remaniements résultent sans doute les "descentes" ou les "remontées" d'outillages préhistoriques dans les matériaux limoneux; ces outillages ont servi de fondement à la stratigraphie locale des limons (H. Breuil 1937), mais celle-ci ne revêt vraisemblablement pas la portée générale et systématique qu'on a voulu lui assigner.

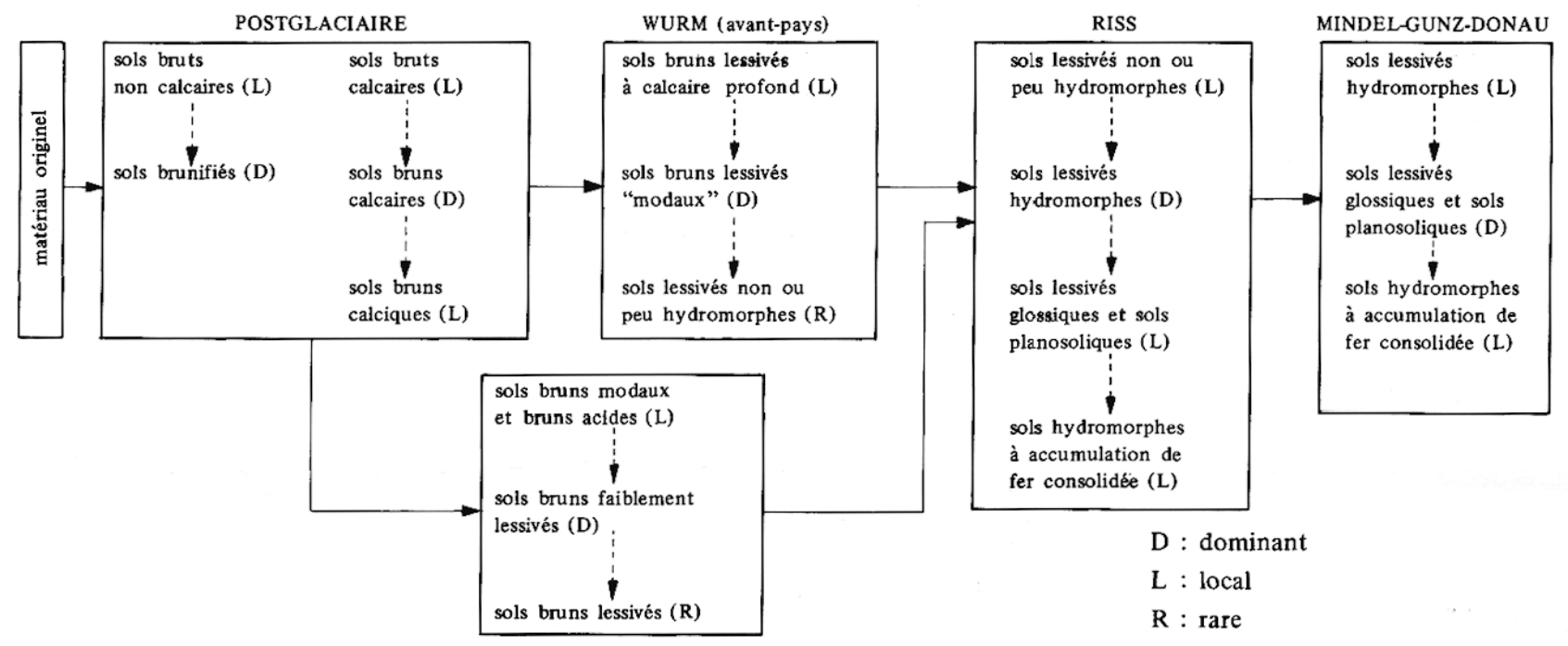

WURM (bordure pyrénéenne) 


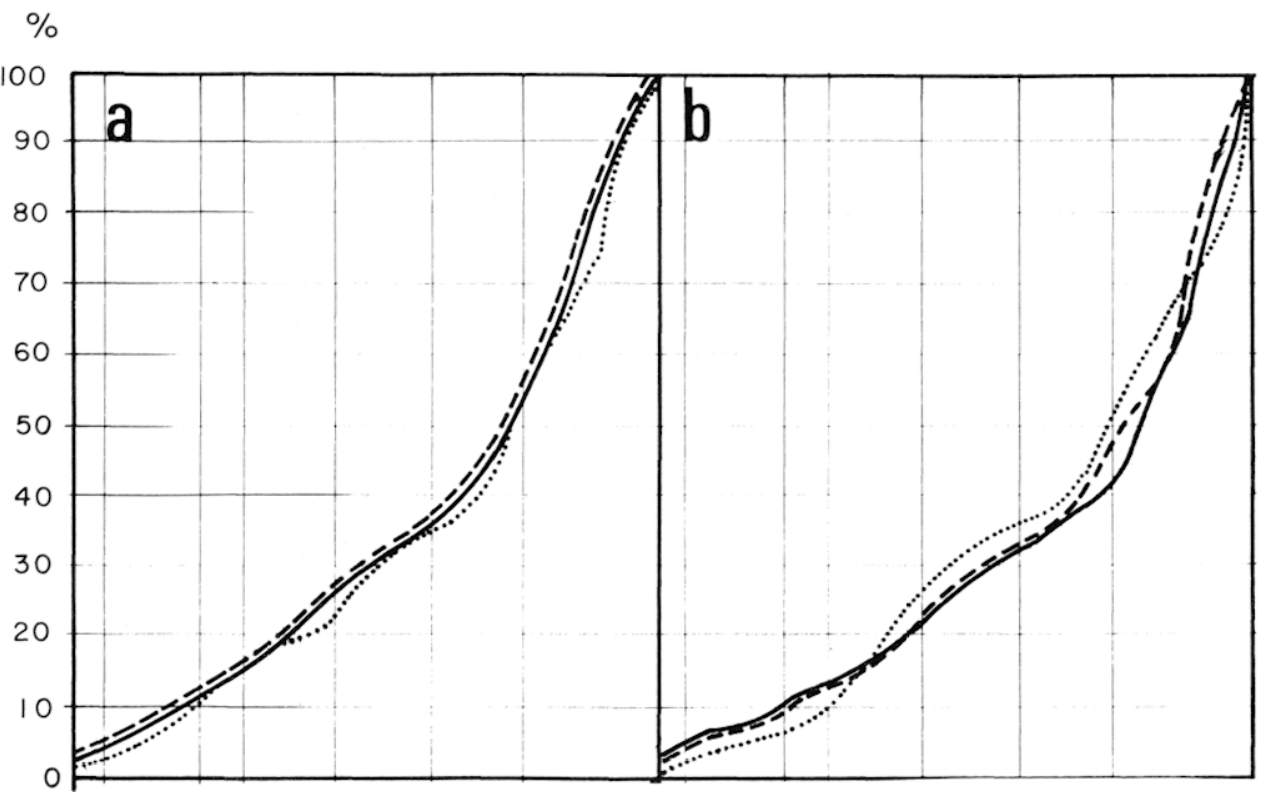

Fig. 17 - Distribution fréquentielle des sables des limons recouvrant les terrasses du Donau. Courbes évoquant plutôt une mise en place fluviatile ou par ruissellement. (Echelle horizontale : logarithme de la dimension des particules, de $0,040 \mathrm{~mm}-\grave{a}$ droite - à $2 \mathrm{~mm}$ ).

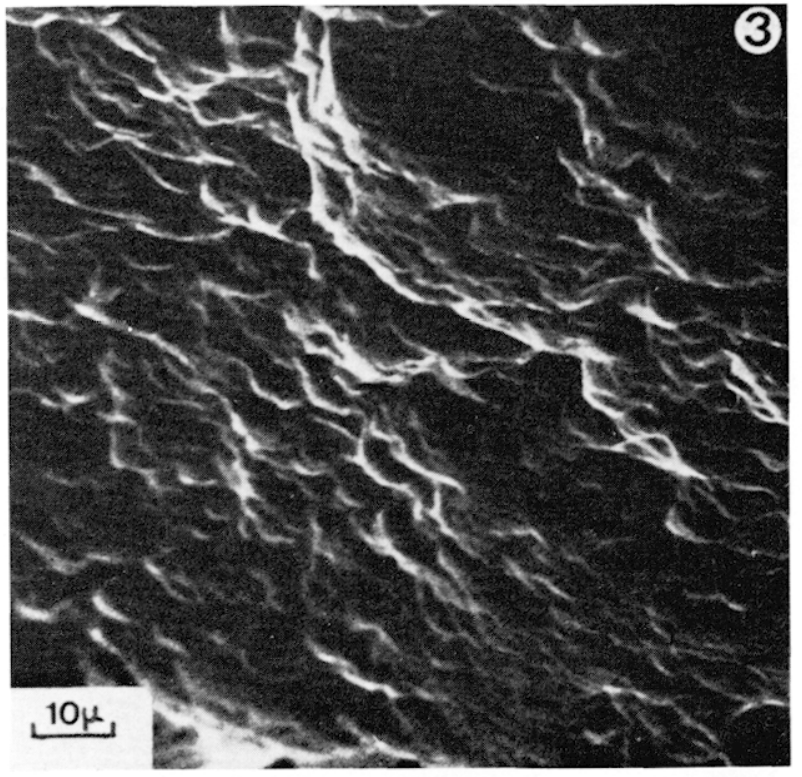

Photo 20 - Modelé de surface des grains de quartz inclus dans les limons du Donau (microscope électronique à balayage). Aspect "en coulées" de la pellicule de silice, avec polissages, caractéristique d'une évolution en milieu fluviatile ou ruisselé.

pas devoir être retenue. Aussi bien les stocks de minéraux lourds, d'origine purement molassique, que les cortèges de minéraux argileux (absence ou rareté des kaolinites), contredisent formellement ce point de vue (J. Hubschman 1975 a).

En définitive, faute d'arguments "éoliens" fiables et jusqu'à preuve du contraire, la majeure partie des couvertures limoneuses qui drapent les terrasses garon-

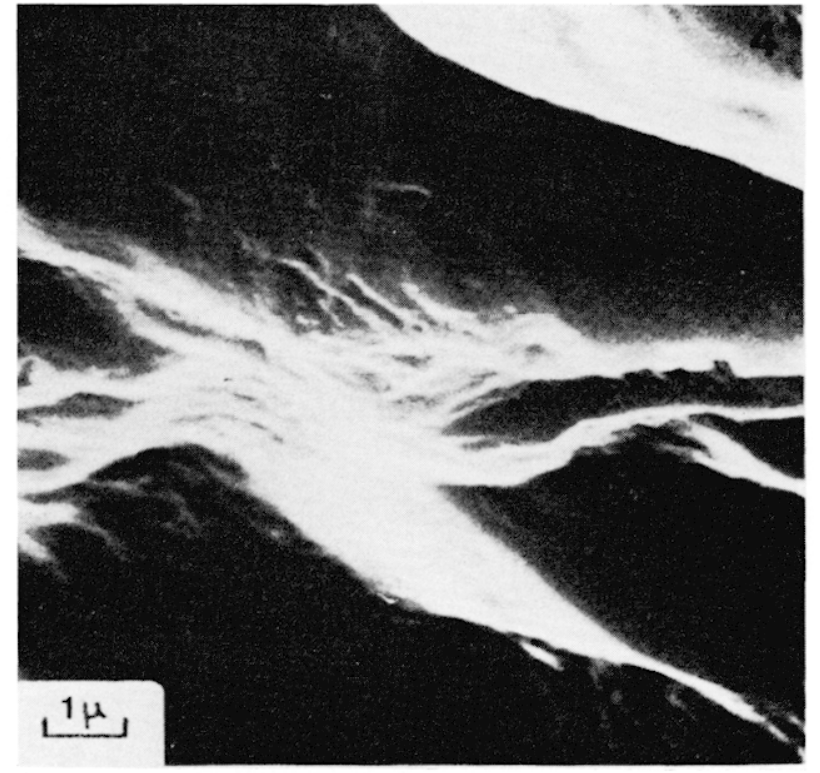

Photo 21 - Grossissement local de la photo 20. Traces de chocs en milieux aqueux, plus ou moins polies.

naises doit être considérée comme d'origine fluviatile (phase terminale de chaque remblaiement) et plus ou moins remaniée par la suite par ruissellement, mouvements de masse ou tout autre phénomène de mobilisation.

\section{Bibliographie}

(Voir p. 214). 